\title{
A general theory for gauge-free lifting
}

\author{
P. J. Morrison* \\ Department of Physics and Institute for Fusion Studies, \\ The University of Texas at Austin, Austin, TX, 78712, USA
}

(Dated: June 25, 2021)

\begin{abstract}
A theory for lifting equations of motion for charged particle dynamics, subject to given electromagnetic like forces, up to a gauge-free system of coupled Hamiltonian Vlasov-Maxwell like equations is given. The theory provides very general expressions for the polarization and magnetization vector fields in terms of the particle dynamics description of matter. Thus, as is common in plasma physics, the particle dynamics replaces conventional constitutive relations for matter. Several examples are considered including the usual Vlasov-Maxwell theory, a guiding center kinetic theory, Vlasov-Maxwell theory with the inclusion of spin, and a Vlasov-Maxwell theory with the inclusion of Dirac's magnetic monopoles. All are shown to be Hamiltonian field theories and the Jacobi identity is proven directly.
\end{abstract}

Key Words: Vlasov, Maxwell, Hamiltonian, noncanonical Poisson bracket, gauge-free, constitutive

\footnotetext{
*morrison@physics.utexas.edu
} 


\section{INTRODUCTION}

In conventional treatments of electricity and magnetism phenomenological susceptibilities are introduced to describe material media. Concomitant with the introduction of these susceptibilities is the idea that charge can be separated into bound and free components, current can be similarly decomposed, and based on these separations expressions for the polarization and magnetization of the medium are obtained. However, it is well-known to plasma physicists that such a simple characterization is not possible for plasmas, where particle orbits may transition from trapped to passing and, indeed, may exhibit complicated behavior that can only be described by the self-consistent treatment of the dynamics of both the particles and the fields. Because of these complications, tractable and reliable expressions for the polarization and magnetization are not so forthcoming, particularly when approximations are made and/or additional physics is added.

The purpose of the present paper is to construct a general theory for the coupling of charge carrying particle dynamics, entities possibly with internal degrees of freedom described by a kinetic theory, coupled to electromagnetic-like field theories. A theory that is gaugefree and ultimately expressible without the introduction of vector and scalar potentials is constructed. Like Maxwell's equations, the Vlasov-Maxwell equations, and virtually every important system in physics, the theory will have Hamiltonian form. This Hamiltonian form will be noncanonical, following the program initiated in Refs. [1, 2].

The construction begins in Sec. II] with a set of ordinary differential equations that describe the particle dynamics. This set of equations, which is the basic model of the matter under consideration, is assumed to have a very general Hamiltonian form, possibly with an unconventional phase space and with a Hamiltonian that depends on specified electromagnetic fields including the field variables $\mathbf{E}(\mathbf{x}, t)$ and $\mathbf{B}(\mathbf{x}, t)$, and possibly all their derivatives. The problem then is to lift this finite-dimensional dynamical system that describes the matter to a gauge-free field theory with a kinetic component that is of Vlasov type coupled to an electromagnetic component of Maxwell type. The difficulty with this lifting program lies in the coupling of the two components of the field theory. It is shown in Sec. III that the construction given naturally results in a field theory that is also Hamiltonian. This assures that there is a consistency to the coupling. Because the Hamiltonian theory requires variational calculus, it is most convenient to discuss constitutive relations resulting from the 
matter system in this section as well. In Sec. IV several examples are presented, beginning with the usual Vlasov-Maxwell system, followed by a general guiding center kinetic theory, a theory that includes spin and, to show the generality of our construction, a theory with monopole charge where the Maxwell field is modified. Gaussian units are used for all examples. Section $\mathrm{V}$ contains concluding remarks. In Appendix $\mathrm{A}$ of the paper there are several subsections with direct proofs of the Jacobi identity for Poisson brackets of the noncanonical Hamiltonian field theories. The first one describes an old calculation of the author that has not heretofore appeared in print, a calculation that contains several useful techniques. The other subsections contain analyses of the other brackets of the examples of Sec. IV]

\section{A GENERAL ELECTROMAGNETIC KINETIC THEORY VIA LIFTING}

Consider a general dynamical system with an $n$-dimensional phase space with coordinates $z=\left(z^{1}, z^{2}, \ldots, z^{n}\right)$ and evolution determined by a Poisson bracket and Hamiltonian $\mathcal{E}$ as follows:

$$
\dot{z}^{a}=\left[z^{a}, \mathcal{E}\right]=J^{a b} \frac{\partial \mathcal{E}}{\partial z^{b}}, \quad a, b=1,2, \ldots, n
$$

where the Poisson bracket on phase space functions $g$ and $h$ is defined by

$$
[g, h]=\frac{\partial g}{\partial z^{a}} J^{a b} \frac{\partial h}{\partial z^{b}}
$$

and repeated indices are to be summed. The only requirements placed on the cosymplectic tensor $J$ is that it endow the Poisson bracket with the properties of antisymmetry, $[g, h]=$ $-[h, g]$, and the Jacobi identity, $[g,[h, k]]+[h,[k, g]]+[k,[g, h]]=0$ for all functions $g, h, k$ (see e.g. [3, 4]). In the context of geometrical mechanics this is referred to as a flow on a Poisson manifold, but this formalism and its language will not be used here. Rather, the physics of matter described by this finite-dimensional dynamical system, as embodied in the Hamiltonian function $\mathcal{E}$, is emphasized. The description of the matter in the formalism of this paper is contained in this function $\mathcal{E}$ and its associated Poisson bracket (2).

Particle orbits in given $\mathbf{E}(\mathbf{x}, t)$ and $\mathbf{B}(\mathbf{x}, t)$ fields are usually described in terms of the electromagnetic potentials, $\phi(\mathbf{x}, t)$ and $\mathbf{A}(\mathbf{x}, t)$, where

$$
\mathbf{E}=-\nabla \phi-\frac{1}{c} \frac{\partial \mathbf{A}}{\partial t} \quad \text { and } \quad \mathbf{B}=\nabla \times \mathbf{A}
$$


Following this usual procedure, the Hamiltonian $\mathcal{E}$ of the general system of (1) will be restricted for the purposes of the present lift theory to have the following form:

$$
\mathcal{E}=\overline{\mathcal{K}}(\mathbf{p}-e \mathbf{A} / c, w, \mathbf{E}, \mathbf{B}, \nabla \mathbf{E}, \nabla \mathbf{B}, \ldots)+e \phi,
$$

where $\overline{\mathcal{K}}$ is an arbitrary function of its arguments. This form was proposed in the context of the variational theory of Refs. [5-7]. Since the aim is to generalize usual charged particle dynamics, the parameter $e$ that denotes charge is included and $c$ is the speed of light as usual. This particular form assures electromagnetic gauge invariance. Here, the phase space has been split into two parts, $z_{p}=\left(\mathbf{x}, \mathbf{p}, w^{1}, w^{2}, \ldots, w^{d}\right)$, where the coordinates $(\mathbf{x}, \mathbf{p})$ are the usual canonical six-dimensional phase space coordinates and the coordinates $w=$ $\left(w^{1}, w^{2}, \ldots, w^{d}\right)$ describe additional degrees of freedom, such as might occur in the classical description of a molecule with rotational or vibrational degrees of freedom. These additional coordinates will be referred to as internal degrees of freedom. An important, but not the only, Poisson bracket is given by

$$
\begin{aligned}
{[f, g] } & =[f, g]_{\mathbf{p}}+[f, g]_{w} \\
& =: \nabla f \cdot \frac{\partial g}{\partial \mathbf{p}}-\nabla g \cdot \frac{\partial f}{\partial \mathbf{p}}+\frac{\partial f}{\partial w^{\alpha}} J_{w}^{\alpha \beta} \frac{\partial g}{\partial w^{\beta}}, \quad \alpha, \beta=1,2, \ldots, d,
\end{aligned}
$$

which is the sum of canonical and internal pieces, and is assumed to satisfy the Jacobi identity. Such a bracket and Hamiltonian generate dynamics of the form of (1).

Several comments on the Hamiltonian form of (44) and (5) are in order. Note that $\mathcal{E}$ may depend explicitly on the fields, and all of their derivatives, and the same may be true for the tensor $J$ of the Poisson bracket provided the Jacobi identity is satisfied for any choice of these fields. The Poisson bracket may also have explicit $w$ dependence, but no direct coupling of the internal degrees of freedom to the fields has been made explicit. In general, such coupling would need to be consistent with the symmetries of interest for these variables. For example, if $w$ were a spin variable, say $\mathbf{s}$, then $\mathcal{E}$ would depend on $\mathbf{s} \cdot \mathbf{B}$. This case is treated as an example in Sec. IVC, Also, observe that all of the dependence on the spatial variable $\mathbf{x}$ occurs through the fields. The omission of such explicit dependence in $\mathcal{E}$ is appropriate for media where spatial homogeneity is broken only by the presence of the fields; however, the $\mathbf{x}$ dependence could be added for further generalization.

Alternatively, a manifestly gauge invariant form is obtained in terms of the coordinates $z_{v}=\left(\mathbf{x}, \mathbf{v}, w^{1}, w^{2}, \ldots, w^{d}\right)$, where $(\mathbf{x}, \mathbf{v})$ denotes the usual six-dimensional velocity phase 
space coordinates, with

$$
\mathbf{p}:=m \mathbf{v}+\frac{e}{c} \mathbf{A}
$$

and $m$ denoting the mass of the charged particle. In terms of these variables (1) takes the form

$$
\dot{z}_{v}=\left[z_{v}, \mathcal{K}\right]+\frac{e}{m} \mathcal{I}_{d} \cdot \mathbf{E},
$$

where the bracket of (5) becomes the Littlejohn [8] Poisson bracket in (77),

$$
[g, h]_{L}=[g, h]_{\mathbf{v}}+[g, h]_{\mathbf{B}}
$$

where

$$
[g, h]_{\mathbf{v}}=\frac{1}{m}\left(\nabla g \cdot \frac{\partial h}{\partial \mathbf{v}}-\nabla h \cdot \frac{\partial g}{\partial \mathbf{v}}\right) \quad \text { and } \quad[g, h]_{\mathbf{B}}=\frac{e}{m^{2} c} \mathbf{B} \cdot\left(\frac{\partial g}{\partial \mathbf{v}} \times \frac{\partial h}{\partial \mathbf{v}}\right)
$$

$\mathcal{I}_{d}$ is a $(6+d) \times 3$ matrix used to embed $\mathbf{E}$ into the force law, and

$$
\mathcal{K}(\mathbf{v}, w ; \mathbf{E}, \mathbf{B}, \nabla \mathbf{E}, \nabla \mathbf{B}, \ldots)=\overline{\mathcal{K}}(\mathbf{p}-e \mathbf{A} / c, w, \mathbf{E}, \mathbf{B}, \nabla \mathbf{E}, \nabla \mathbf{B}, \ldots)
$$

Thus the electric field appears as an external force in addition to any dependence on it that may come through the function $\mathcal{K}$, and the electromagnetic potentials no longer appear in the dynamics. Note, in general $\mathbf{E}$ cannot be written as a gradient in order to combine it with the first term of (7). The dynamics of (7) with arbitrary Poisson bracket in terms of $z_{v}$, possibly depending explicitly on $z_{v}, \mathbf{E}$, and $\mathbf{B}$, can be taken as the starting point for lifting to a kinetic theory.

Usual Lorentzian dynamics is given by $\mathcal{K}=m|\mathbf{v}|^{2} / 2$ : when $\mathcal{K}$ is written in terms of $\mathbf{p}$, the bracket of (5) with $\mathcal{E}$ yields the equations of motion for a particle of charge $e$ and mass $m$ subject to given electric and magnetic fields. Alternatively, the same equations are given from (77) with $[,]_{L}$.

Now, the finite degree-of-freedom system of (7) will be lifted. The first step is to lift the particle dynamics to a kinetic theory for determining a phase space density $f(z, t)=$ $f(\mathbf{x}, \mathbf{v}, w, t)$. This is easily achieved by the standard Liouville form

$$
\frac{\partial f}{\partial t}+[f, \mathcal{K}]+\frac{e}{m} \mathbf{E} \cdot \frac{\partial f}{\partial \mathbf{v}}=0
$$

where the generalization to multiple species is straightforward. Clearly the characteristic equations of (11) correspond to the finite-dimensional matter model of (7). 
The second part of lifting is to describe the coupling to Maxwell's equations. This coupling is effected by introducing the energy functional

$$
K[\mathbf{E}, \mathbf{B}, f]:=\int d \mathbf{x} d \mathbf{v} d w \mathcal{K} f
$$

whence the following expressions for the charge and current densities are obtained:

$$
\begin{aligned}
& \rho(\mathbf{x}, t)=e \int d \mathbf{v} d w f-\nabla \cdot \frac{\delta K}{\delta \mathbf{E}} \\
& \mathbf{J}(\mathbf{x}, t)=e \int d \mathbf{v} d w \frac{\partial \mathcal{K}}{\partial \mathbf{v}} f+\frac{\partial}{\partial t} \frac{\delta K}{\delta \mathbf{E}}+c \nabla \times \frac{\delta K}{\delta \mathbf{B}} .
\end{aligned}
$$

Inserting these expressions for the sources into the usual form of Maxwell's equations completes the lift.

From (13) and (14) it is evident that the polarization, $\mathbf{P}$, and magnetization, $\mathbf{M}$, can be identified as

$$
\mathbf{P}(\mathbf{x}, t)=-\frac{\delta K}{\delta \mathbf{E}} \quad \text { and } \quad \mathbf{M}(\mathbf{x}, t)=-\frac{\delta K}{\delta \mathbf{B}}
$$

which is consistent with the usual definitions of bound charge density, polarization current, and magnetization current,

$$
\rho_{b}=-\nabla \cdot \mathbf{P}, \quad \mathbf{J}_{p}=\frac{\partial \mathbf{P}}{\partial t}, \quad \text { and } \quad \mathbf{J}_{m}=c \nabla \times \mathbf{M}
$$

respectively. Although the manner of lifting embodied in (11), (13), and (14) is straightforward, because of the functional derivatives in (15) the dependencies of $\mathbf{P}$ and $\mathbf{M}$ on the fields $\mathbf{E}$ and $\mathbf{B}$ may be very complicated and contain high order spatial derivatives.

In Sec. III it is shown that this manner of lifting results in a Hamiltonian field description of the coupled system. It should be emphasized that this construction does not require the explicit introduction of the vector and scalar potentials. However, from the Hamiltonian form using $\mathcal{E}$ it is clear that it subsumes the description using $\phi(\mathbf{x}, t)$ and $\mathbf{A}(\mathbf{x}, t))$. To see the explicit form, define the momentum phase space density by $\bar{f}(\mathbf{x}, \mathbf{p}, w, t)=f(\mathbf{x}, \mathbf{v}, w, t)$, which gives under the change $\mathbf{v} \leftrightarrow \mathbf{p}$ the governing kinetic equation

$$
\frac{\partial \bar{f}}{\partial t}+[\bar{f}, \mathcal{E}]=0
$$

with $[g, h]=[f, g]_{\mathbf{p}}+[f, g]_{w}$. The coupling to Maxwell's equation is essentially unchanged:

$$
\begin{aligned}
\rho(\mathbf{x}, t) & =e \int d \mathbf{p} d w \bar{f}-\nabla \cdot \frac{\delta K}{\delta \mathbf{E}} \\
\mathbf{J}(\mathbf{x}, t) & =e \int d \mathbf{p} d w \frac{\partial \mathcal{K}}{\partial \mathbf{p}} \bar{f}+\frac{\partial}{\partial t} \frac{\delta K}{\delta \mathbf{E}}+c \nabla \times \frac{\delta K}{\delta \mathbf{B}},
\end{aligned}
$$


as are the expressions for $\mathbf{P}$ and $\mathbf{M}$. Using the chain rule expressions,

$$
\frac{\partial \bar{f}}{\partial \mathbf{p}}=\frac{1}{m} \frac{\partial f}{\partial \mathbf{v}}, \quad \nabla \bar{f}=\nabla f-\frac{e}{m c} \frac{\partial f}{\partial \mathbf{v}} \cdot \nabla \mathbf{A}, \quad \text { and } \quad \frac{\partial \bar{f}}{\partial t}=\frac{\partial f}{\partial t}-\frac{e}{m c} \frac{\partial f}{\partial \mathbf{v}} \cdot \frac{\partial \mathbf{A}}{\partial t},
$$

it is not difficult to show that $[g, h]_{\mathbf{p}}$ transforms to $[g, h]_{L}$ and (17) transforms into (11).

\section{HAMILTONIAN FORM AND CONSTITUTIVE RELATIONS}

Based on past experience, viz. Vlasov-Maxwell and guiding center kinetic theories, a natural choice for the Hamiltonian functional is the following:

$$
\begin{aligned}
H[f, \mathbf{E}, \mathbf{B}] & =K-\int d \mathbf{x} \mathbf{E} \cdot \frac{\delta K}{\delta \mathbf{E}}+\frac{1}{8 \pi} \int d \mathbf{x}\left(|\mathbf{E}|^{2}+|\mathbf{B}|^{2}\right) \\
& =K+\int d \mathbf{x} \mathbf{E} \cdot \mathbf{P}+\frac{1}{8 \pi} \int d \mathbf{x}\left(|\mathbf{E}|^{2}+|\mathbf{B}|^{2}\right)
\end{aligned}
$$

where $\mathbf{P}$, as given by (15), is used as a shorthand in the second line, which one could rewrite in terms of $\mathbf{D}:=\mathbf{E}+4 \pi \mathbf{P}$. The Hamiltonian of (21) is a generalization of the energy component of the energy-momentum tensor first derived by variational methods in [5-7]. It is straightforward to verify directly that (21) is conserved by the combined field theory, Maxwell's equations with the sources (13) and (14) coupled to the kinetic theory of (11).

One might think that the $|\mathbf{B}|^{2}$ term of (21) should be replaced by $\mathbf{B} \cdot \mathbf{H}$, where $\mathbf{H}=$ $\mathbf{B}-4 \pi \mathbf{M}$, but this is incorrect. All polarization and magnetization effects are modeled here by the terms involving $\mathcal{K}$, i.e., they are a consequence of the particle dynamics. Rather than relating $\mathbf{E}$ and $\mathbf{B}$ to $\mathbf{D}$ and $\mathbf{H}$ by constitutive relations, the particle dynamics, extended and other, describes the physics that is often approximated by simplistic constitutive relations. For example, the difference between $|\mathbf{E}|^{2}$ and $\mathbf{E} \cdot \mathbf{D}$ arises from the $\mathcal{K}$ term that contains the matter dynamical information.

If only $(f, \mathbf{E}, \mathbf{B})$ are used as dynamical variables, there is a difficulty in obtaining a Poisson bracket description for the field theory. The problem is readily encountered when one attempts to include polarization effects, because the polarization current has a time derivative and Poisson bracket expressions such as $\{\mathbf{E}, H\}$ do not produce terms with time derivatives of the dynamical variables; i.e., in Hamiltonian theories all time derivatives are on the left hand side, so to speak. Consequently a term of the form $\partial \mathbf{P} / \partial t$ cannot appear. However, there is a way to circumvent this problem, a problem that does not occur in action principle formulations, such as those of [5 7]. 
Functional differentiation of (21) gives

$$
\begin{aligned}
& \frac{\delta H}{\delta \mathbf{E}}=-\left(\frac{\delta^{2} K}{\delta \mathbf{E} \delta \mathbf{E}}\right)^{\dagger} \cdot \mathbf{E}+\frac{\mathbf{E}}{4 \pi}, \\
& \frac{\delta H}{\delta \mathbf{B}}=\frac{\delta K}{\delta \mathbf{B}}-\left(\frac{\delta^{2} K}{\delta \mathbf{B} \delta \mathbf{E}}\right)^{\dagger} \cdot \mathbf{E}+\frac{\mathbf{B}}{4 \pi},
\end{aligned}
$$

where $\delta^{2} K / \delta \mathbf{E} \delta \mathbf{E}$ and $\delta^{2} K / \delta \mathbf{E} \delta \mathbf{B}$ are second functional derivative operators that satisfy

$$
\left(\frac{\delta^{2} K}{\delta \mathbf{E} \delta \mathbf{E}}\right)^{\dagger}=\frac{\delta^{2} K}{\delta \mathbf{E} \delta \mathbf{E}} \quad \text { and } \quad\left(\frac{\delta^{2} K}{\delta \mathbf{B} \delta \mathbf{E}}\right)^{\dagger}=\frac{\delta^{2} K}{\delta \mathbf{E} \delta \mathbf{B}}
$$

(See e.g. [3, 4, 9] for a review of functional differentiation.) Expressions (22) and (23) reveal how polarization and magnetization effects are embodied in $\mathcal{K}$. Since the functional derivatives above have, in a sense, 'dressed' $\mathbf{E}$ and $\mathbf{B}$, existing Hamiltonian structures will not be adequate. It is clear that without some modification one cannot obtain the polarization current.

If the theory were expressed in terms of $\mathbf{D}$, the following bracket on functionals $\bar{F}[\mathbf{D}, \mathbf{B}, f]$ could give the correct temporal evolution of $\mathbf{D}$, provided $\delta \bar{H} / \delta \mathbf{B}=\mathbf{H} / 4 \pi$ :

$$
\begin{aligned}
\{\bar{F}, \bar{G}\}= & \int d \mathbf{x} d \mathbf{v} d w f\left(\left[\bar{F}_{f}, \bar{G}_{f}\right]_{\mathbf{v}}+\left[\bar{F}_{f}, \bar{G}_{f}\right]_{w}\right) \\
& +\int d \mathbf{x} d \mathbf{v} d w f\left[\bar{F}_{f}, \bar{G}_{f}\right]_{\mathbf{B}} \\
& \quad+\frac{4 \pi e}{m} \int d \mathbf{x} d \mathbf{v} d w f\left(\bar{G}_{\mathbf{D}} \cdot \partial_{\mathbf{v}} \bar{F}_{f}-\bar{F}_{\mathbf{D}} \cdot \partial_{\mathbf{v}} \bar{G}_{f}\right) \\
& +4 \pi c \int d \mathbf{x}\left(\bar{F}_{\mathbf{D}} \cdot \nabla \times \bar{G}_{\mathbf{B}}-\bar{G}_{\mathbf{D}} \cdot \nabla \times \bar{F}_{\mathbf{B}}\right),
\end{aligned}
$$

with $[f, g]_{\mathbf{v}}$ and $[f, g]_{\mathbf{B}}$ given by (9),$\partial_{\mathbf{v}} g=\partial g / \partial \mathbf{v}$, and $\bar{F}_{f}:=\delta \bar{F} / \delta f, \bar{F}_{\mathbf{D}}:=\delta \bar{F} / \delta \mathbf{D}$, etc. The Born-Infeld term of (28) is motivated by their original theory [10] that was also written in terms of $\mathbf{D}$ and $\mathbf{B}$ (see also [11]). Although this term can give something like $\partial \mathbf{D} / \partial t=\nabla \times \mathbf{H}$, it remains to properly define the meaning of $\mathbf{D}$ and $\mathbf{H}$. Thus, this bracket alone does not constitute a closed theory. Similarly the coupling term (27), a generalization of that introduced in [2, 3] that includes the internal variable $w$, is written here in terms of $\mathbf{D}$, but the generalization of the Marsden-Weinstein term [12] (see also [13]) of (26]), and the first term of (25), also a generalization of that given in [2, 3], are unchanged. The new internal term here of (25) with $\left[\bar{F}_{f}, \bar{G}_{f}\right]_{w}$ does not depend on $\mathbf{D}$ and does not affect the Jacobi identity (cf. Appendix A 2). 
To close the theory requires a constitutive relation, something like $\mathbf{D}=\underline{\underline{\epsilon}} \cdot \mathbf{E}$. Such relations are often appended to electromagnetic theory based on phenomenological material properties, but here they emerge as a consequence of the Vlasov-like dynamics and the definitions (15). Using (15) gives

$$
\mathbf{D}=\mathbf{D}[\mathbf{E}, \mathbf{B} ; f]=\mathbf{E}+4 \pi \mathbf{P}[\mathbf{E}, \mathbf{B} ; f]
$$

with both $\mathbf{P}$ and $\mathbf{D}$ linear in $f$, but not in $\mathbf{E}$ and $\mathbf{B}$. In general these functionals can be nonlinear and even global in nature. It is only required that there be a unique inverse

$$
\mathbf{E}=\mathbf{D}^{-1}[\mathbf{D}, \mathbf{B} ; f]=\mathbf{E}[\mathbf{D}, \mathbf{B} ; f] .
$$

Similarly, using (15)

$$
\mathbf{H}=\mathbf{H}[\mathbf{B}, \mathbf{E} ; f]=\mathbf{B}-4 \pi \mathbf{M}[\mathbf{B}, \mathbf{E} ; f],
$$

which is also assumed to have an inverse, i.e.

$$
\mathbf{B}=\mathbf{B}[\mathbf{H}, \mathbf{E} ; f]=\mathbf{H}+4 \pi \mathbf{M}[\mathbf{H}, \mathbf{E} ; f]
$$

For given $\mathcal{K}$, the expressions of (15) can be quite complicated, particularly when derivatives of the fields are included. However, these expressions are local in time, i.e., E, B, D, and $f$ are all evaluated at the same time. Because of the presence of $f$ and the equation governing it, causal effects are included in a dynamical sense and do not need to be put in at the expense of breaking time-reversal symmetry. Also, there is no artificial separation of charge into bound and free components or current into magnetization or other. Rather, charges and currents are determined dynamically according to the Vlasov equation. A given charge may behave in any manner consistent with this dynamics.

When $\lim _{\mathbf{E} \rightarrow 0} \mathbf{D}[\mathbf{E}, \mathbf{B} ; f]=0$ the first order term in an expansion in $\mathbf{E}$ gives $\mathbf{D}=\underline{\underline{\epsilon}} \cdot$ E, where $\underline{\underline{\epsilon}}$ is the dielectric permittivity operator. Similarly, $\mathbf{B}=\underline{\underline{\mu}} \cdot \mathbf{H}$, where $\underline{\underline{\mu}}$ is the permeability operator. If one were to replace the Vlasov dynamics by trivial dynamics of linear response away from equilibrium, then one can recover the usual permittivity and permeability relations, including the usual causal (see e.g. [14]) form in space and time. But, this will not be done here.

To sum up, the Hamiltonian of (21) is given in terms of $(\mathbf{E}, \mathbf{B}, f)$, the bracket of (25)-(28) in terms of $(\mathbf{D}, \mathbf{B}, f)$, and (29) is a closure relation relating $\mathbf{D}$ to $\mathbf{E}$. Thus, if $\bar{H}[\mathbf{D}, \mathbf{B}, f]=$ $H[\mathbf{E}, \mathbf{B}, f]$ is defined by inserting the inverse of $(29)$ in for $\mathbf{E}$, a closed theory is obtained. 
For general $\mathcal{K}$ this inversion cannot be done explicitly (although a series expansion may be possible); however, the chain rule can be used to relate functional derivatives of $H$ to those of $\bar{H}$ and thereby obtain equations for the time derivatives of $(\mathbf{D}, \mathbf{B}, f)$ which can then be shown to be equivalent to those of Sec. II. Alternatively, the chain rule can be used to write the bracket of (25)-(28) in terms of the set of fundamental variables $(\mathbf{E}, \mathbf{B}, f)$.

To understand the chain rule, suppose two functionals are related by $\bar{F}[\mathbf{D}, \mathbf{B}, f]=$ $F[\mathbf{E}, \mathbf{B}, f]$, where the right hand side is obtained by inserting (29) in for $\mathbf{D}$ in the functional $F$ on the left. Variation of $\bar{F}=F$ gives

$$
\int d \mathbf{x}\left(\frac{\delta \bar{F}}{\delta \mathbf{D}} \cdot \delta \mathbf{D}+\frac{\delta \bar{F}}{\delta \mathbf{B}} \cdot \delta \mathbf{B}\right)+\int d z \frac{\delta \bar{F}}{\delta f} \delta f=\int d \mathbf{x}\left(\frac{\delta F}{\delta \mathbf{E}} \cdot \delta \mathbf{E}+\frac{\delta F}{\delta \mathbf{B}} \cdot \delta \mathbf{B}\right)+\int d z \frac{\delta F}{\delta f} \delta f,
$$

while variation of $(\underline{30})$ gives

$$
\delta \mathbf{E}=\frac{\delta \mathbf{E}}{\delta \mathbf{D}} \cdot \delta \mathbf{D}+\frac{\delta \mathbf{E}}{\delta \mathbf{B}} \cdot \delta \mathbf{B}+\frac{\delta \mathbf{E}}{\delta f} \delta f,
$$

where $\delta \mathbf{E} / \delta \mathbf{D}$ etc. are the usual Fréchet derivatives obtained by first variation. Inserting (34) into (33) and comparing the coefficients of the independent variations $\delta \mathbf{D}$ etc., gives

$$
\begin{aligned}
& \frac{\delta \bar{F}}{\delta \mathbf{D}}=\left(\frac{\delta \mathbf{E}}{\delta \mathbf{D}}\right)^{\dagger} \cdot \frac{\delta F}{\delta \mathbf{E}}, \\
& \frac{\delta \bar{F}}{\delta \mathbf{B}}=\frac{\delta F}{\delta \mathbf{B}}+\left(\frac{\delta \mathbf{E}}{\delta \mathbf{B}}\right)^{\dagger} \cdot \frac{\delta F}{\delta \mathbf{E}}, \\
& \frac{\delta \bar{F}}{\delta f}=\frac{\delta F}{\delta f}+\left(\frac{\delta \mathbf{E}}{\delta f}\right)^{\dagger} \cdot \frac{\delta F}{\delta \mathbf{E}} .
\end{aligned}
$$

A more explicit expression for $(\delta \mathbf{E} / \delta \mathbf{D})^{\dagger}$ can be obtained by varying (29) at fixed $\mathbf{B}$ and $f$, giving

$$
\delta \mathbf{D}=\left(\underline{\underline{I}}-4 \pi \frac{\delta^{2} K}{\delta \mathbf{E} \delta \mathbf{E}}\right) \cdot \delta \mathbf{E}=: \underline{\underline{\varepsilon}} \cdot \delta \mathbf{E}
$$

or

$$
\delta \mathbf{E}=\frac{\delta \mathbf{E}}{\delta \mathbf{D}} \cdot \delta \mathbf{D}=\underline{\underline{\varepsilon}}^{-1} \cdot \delta \mathbf{D}
$$

where $\underline{\underline{\varepsilon}}$ is the nonlinear permittivity operator (not to be confused with $\underline{\underline{\epsilon}}$ ). Evidently,

$$
\mathbf{E}_{\mathbf{D}}:=\frac{\delta \mathbf{E}}{\delta \mathbf{D}}=\underline{\underline{\varepsilon}}^{-1}=\left(\underline{\underline{I}}-4 \pi \frac{\delta^{2} K}{\delta \mathbf{E} \delta \mathbf{E}}\right)^{-1}
$$

and $\mathbf{E}_{\mathbf{D}}=\left(\mathbf{E}_{\mathbf{D}}\right)^{\dagger}$ or $\left(\underline{\underline{\varepsilon}}^{-1}\right)^{\dagger}=\underline{\underline{\varepsilon}}^{-1}$. It is important to note that although $\delta \mathbf{D}=\underline{\underline{\varepsilon}} \cdot \delta \mathbf{E}$, $\mathbf{D} \neq \underline{\underline{\varepsilon}} \cdot \mathbf{E}$; the correct relation between $\mathbf{D}$ and $\mathbf{E}$ is given by the nonlinear expression of (29) $)$. Similarly,

$$
\mathbf{E}_{\mathbf{B}}:=\frac{\delta \mathbf{E}}{\delta \mathbf{B}}=4 \pi \underline{\underline{\varepsilon}}^{-1} \cdot \frac{\delta^{2} K}{\delta \mathbf{B} \delta \mathbf{E}} \quad \text { and } \quad \mathbf{E}_{f}:=\frac{\delta \mathbf{E}}{\delta f}=4 \pi \underline{\underline{\varepsilon}}^{-1} \cdot \frac{\delta^{2} K}{\delta f \delta \mathbf{E}}
$$


and the functional derivatives of (35)-(37) can now be calculated:

$$
\begin{aligned}
\bar{H}_{\mathbf{D}} & =\mathbf{E}_{\mathbf{D}}^{\dagger} \cdot H_{\mathbf{E}}=\underline{\underline{\varepsilon}}^{-1} \cdot \underline{\underline{\varepsilon}} \cdot \frac{\mathbf{E}}{4 \pi} \\
& =\frac{\mathbf{E}}{4 \pi} \\
\bar{H}_{\mathbf{B}} & =H_{\mathbf{B}}+\mathbf{E}_{\mathbf{B}}^{\dagger} \cdot H_{\mathbf{E}}=K_{\mathbf{B}}-\left(\frac{\delta^{2} K}{\delta \mathbf{B} \delta \mathbf{E}}\right)^{\dagger} \cdot \mathbf{E}+\frac{\mathbf{B}}{4 \pi}+\left(\underline{\underline{\varepsilon}}^{-1} \cdot \frac{\delta^{2} K}{\delta \mathbf{B} \delta \mathbf{E}}\right)^{\dagger} \cdot \underline{\underline{\varepsilon}} \cdot \mathbf{E} \\
& =\frac{\mathbf{B}}{4 \pi}-\mathbf{M} \\
\bar{H}_{f} & =H_{f}+\mathbf{E}_{f}^{\dagger} \cdot H_{\mathbf{E}}=\mathcal{K}-\left(\frac{\delta^{2} K}{\delta f \delta \mathbf{E}}\right)^{\dagger} \cdot \mathbf{E}+\left(\underline{\underline{\varepsilon}}^{-1} \cdot \frac{\delta^{2} K}{\delta f \delta \mathbf{E}}\right)^{\dagger} \cdot \underline{\underline{\varepsilon}} \cdot \mathbf{E} \\
& =\mathcal{K} .
\end{aligned}
$$

Now, using the expressions of (42) in the bracket of (25) $-(28)$ gives

$$
\begin{aligned}
\frac{\partial \mathbf{B}}{\partial t} & =-4 \pi c \nabla \times \bar{H}_{\mathbf{D}}=-c \nabla \times \mathbf{E} \\
\frac{\partial \mathbf{D}}{\partial t} & =4 \pi c \nabla \times \bar{H}_{\mathbf{B}}-\frac{4 \pi e}{m} \int d \mathbf{v} d w f \partial_{\mathbf{v}} \bar{H}_{f} \\
& =c \nabla \times \mathbf{H}-\frac{4 \pi e}{m} \int d \mathbf{v} d w f \partial_{\mathbf{v}} \mathcal{K} \\
\frac{\partial f}{\partial t} & =-\left[f, \bar{H}_{f}\right]-\partial_{\mathbf{v}} \cdot\left(f \bar{H}_{\mathbf{D}}\right) \\
& =-[f, \mathcal{K}]-\frac{e}{m} \mathbf{E} \cdot \frac{\partial f}{\partial \mathbf{v}}
\end{aligned}
$$

where $[]=,[,]_{L}+[,]_{w}$ as defined by (8) and (9). Thus the bracket reproduces the Vlasov-like equation of (11) and Maxwell's equations with the polarization and magnetization currents, but remember $f$ and [, ] can be written in terms of $\mathbf{p}$ using (6) and thus the above is also equivalent to (11).

In the above, $\mathbf{D}$ is a convenience, a shorthand for $\mathbf{E}+4 \pi \mathbf{P}$, with $\mathbf{E}$ being the fundamental variable. One could eliminate $\mathbf{D}$ from these equations e.g. by writing (44) as follows:

$$
\frac{\partial \mathbf{D}}{\partial t}=\mathbf{E}_{\mathbf{D}} \cdot \frac{\partial \mathbf{E}}{\partial t}+\mathbf{P}_{\mathbf{B}} \cdot \frac{\partial \mathbf{B}}{\partial t}+\mathbf{P}_{f} \cdot \frac{\partial f}{\partial t}
$$

where $\mathbf{E}_{\mathbf{D}}=\underline{\underline{\varepsilon}}$, as before, and $\mathbf{P}_{\mathbf{B}}$ and $\mathbf{P}_{f}$ are again operators obtained by variation of $\mathbf{P}$, and then inserting the other two equations of motion for the time derivatives. This procedure will lead to a complicated set of equations in terms of the fundamental variables $(\mathbf{E}, \mathbf{B}, f)$. Another way of obtaining these complicated equations is to obtain a bracket in terms of $\mathbf{E}, \mathbf{B}$, and $f$ alone, by inserting the transformations for the functional derivatives of (35), 
(36), and (37) into the bracket of (25)-(28). This yields the following complicated bracket:

$$
\begin{gathered}
\{F, G\}=\int d \mathbf{x} d \mathbf{v} d w f\left[F_{f}+\mathbf{E}_{f}^{\dagger} \cdot F_{\mathbf{E}}, G_{f}+\mathbf{E}_{f}^{\dagger} \cdot G_{\mathbf{E}}\right] \\
+\frac{4 \pi e}{m} \int d \mathbf{x} d \mathbf{v} d w f\left(\left(\mathbf{E}_{\mathbf{D}}^{\dagger} \cdot G_{\mathbf{E}}\right) \cdot \partial_{\mathbf{v}}\left(F_{f}+\mathbf{E}_{f}^{\dagger} \cdot F_{\mathbf{E}}\right)-\left(\mathbf{E}_{\mathbf{D}}^{\dagger} \cdot F_{\mathbf{E}}\right) \cdot \partial_{\mathbf{v}}\left(G_{f}+\mathbf{E}_{f}^{\dagger} \cdot G_{\mathbf{E}}\right)\right) \\
\quad+4 \pi c \int d \mathbf{x}\left(\left(\mathbf{E}_{\mathbf{D}}^{\dagger} \cdot F_{\mathbf{E}}\right) \cdot \nabla \times\left(G_{\mathbf{B}}+\mathbf{E}_{\mathbf{B}}^{\dagger} \cdot G_{\mathbf{E}}\right)\right. \\
\left.\quad-\left(\mathbf{E}_{\mathbf{D}}^{\dagger} \cdot G_{\mathbf{E}}\right) \cdot \nabla \times\left(F_{\mathbf{B}}+\mathbf{E}_{\mathbf{B}}^{\dagger} \cdot F_{\mathbf{E}}\right)\right)
\end{gathered}
$$

where to complete the procedure the expressions of (40) and (41) are to be inserted. Thus the bracket of (47) is quite complicated with many terms and operators. Nevertheless, by its construction it satisfies the Jacobi identity (modulo the $\nabla \cdot \mathbf{B}=0$ obstruction discussed in Appendix A 1). With Hamiltonian $H[\mathbf{E}, \mathbf{B}, f]$ it is easily seen from (42) that this bracket produces the correct equations for $\partial f / \partial t$ and $\partial \mathbf{B} / \partial t$, but it is less easy, yet possible, to see it produces the complications of (46) correctly.

For some theories of interest, including usual linear response theory, $\mathcal{K}$ has a simplified form, viz.

$$
\mathcal{K}(\mathbf{v}, w, \mathbf{E}, \mathbf{B})=h(\mathbf{v}, w, \mathbf{B})+\mathcal{P}(\mathbf{v}, w, \mathbf{B}) \cdot \mathbf{E}+\frac{1}{2} \mathbf{E} \cdot \underline{\underline{k}}(\mathbf{v}, w, \mathbf{B}) \cdot \mathbf{E},
$$

where $\underline{\underline{k}}^{\dagger}=\underline{\underline{k}}$. For example, such a linear polarization theory is sufficient for some drift and gyrokinetic-like theories. With (48)

$$
\mathbf{P}=-\int d \mathbf{v} d w \frac{\partial \mathcal{K}}{\partial \mathbf{E}} f=-\int d \mathbf{v} d w \mathcal{P} f-\mathbf{E} \cdot \int d \mathbf{v} d w \underline{\underline{k}} f .
$$

The first term of the second equality of (49) represents a permanent dipole moment per unit volume, which can be dropped: because (21) is a Legendre transform it will cancel out anyway. The second term of (49) defines the electric susceptibility, $\underline{\underline{\chi_{e}}}$. Thus $\mathbf{P}=\underline{\underline{\chi_{e}}} \cdot \mathbf{E}$ or

$$
\mathbf{D}=\underline{\underline{\epsilon}} \cdot \mathbf{E}
$$

with $\underline{\underline{\epsilon}}=\underline{\underline{I}}+4 \pi \underline{\underline{\chi_{e}}}$. Note that unlike the $\underline{\underline{\varepsilon}}$ of (38), when (48) is assumed, $\underline{\underline{\epsilon}}$ is independent of $\mathbf{E}$ but not of $\mathbf{B}$. Explicitly in terms of indices

$$
\epsilon_{i j}=\delta_{i j}-4 \pi \int d \mathbf{v} d w k_{i j}(\mathbf{v}, w, \mathbf{B}) f .
$$

Assuming $\underline{\underline{\epsilon}}^{-1}$ exists and has the form

$$
\underline{\underline{\epsilon}}^{-1}=\frac{\underline{\underline{I}}}{\underline{\underline{I}}+4 \pi \underline{\underline{\chi_{e}}}}=\underline{\underline{I}}-4 \pi \underline{\underline{\chi_{e}}}+\left(4 \pi \underline{\underline{\chi_{e}}}\right)^{2}-\ldots
$$


where $\underline{\underline{\chi_{e}}}{ }^{2}$ stands for matrix multiplication, and so on down the line. Hence, $\mathbf{E}=\underline{\underline{\epsilon}}^{-1} \cdot \mathbf{D}$. Although $\underline{\underline{\epsilon}}$ is linear in $f$, this is not the case for $\underline{\underline{\epsilon}}^{-1}$. Finally, for the $\mathcal{K}$ of (48) that is quadratic in $\mathbf{E}$, the following simplified expressions are obtained:

$$
\begin{aligned}
& \mathbf{E}_{\mathbf{D}}^{\dagger}=\underline{\underline{\epsilon}}^{-1} \\
& \mathbf{E}_{\mathbf{B}}^{\dagger}=\mathbf{D} \cdot \frac{\partial \underline{\underline{\epsilon}}^{-1}}{\partial \mathbf{B}} \\
& \mathbf{E}_{f}^{\dagger}=\mathbf{D} \cdot \underline{\underline{\epsilon}}^{-1} \cdot \underline{\underline{k}} \cdot \underline{\underline{\epsilon}}^{-1} .
\end{aligned}
$$

Note, obtaining these formulas can be facilitated by using identities obtained by varying the expression $\underline{\underline{\epsilon}}^{-1} \cdot \underline{\underline{\epsilon}}=\underline{\underline{I}}$.

\section{EXAMPLES}

In this section four examples are given: that of Sec. IVA is the usual Vlasov-Maxwell theory, that of Sec. IVB is a guiding center drift kinetic theory that includes nontrivial polarization and magnetization effects, that of Sec. IVC includes a physically perspicuous internal variable, and that of Sec. IVD was chosen to show the generality of the lift theory by altering Maxwell's equations.

\section{A. Vlasov-Maxwell}

For Vlasov-Maxwell theory $w$ is nonexistent and only $z=(\mathbf{x}, \mathbf{p})$ appears. Thus, $\bar{f}(\mathbf{x}, \mathbf{p}, t)$ and, with $\mathcal{K}=|\mathbf{p}-e \mathbf{A} / c|^{2} / 2 m$, Eq. (11) becomes

$$
\begin{aligned}
\frac{\partial \bar{f}}{\partial t} & =\left[\frac{|\mathbf{p}-e \mathbf{A} / c|^{2}}{2 m}+e \phi, \bar{f}\right] \\
& =-\frac{e}{m c}(\mathbf{p}-e \mathbf{A} / c) \cdot \nabla \mathbf{A} \cdot \frac{\partial \bar{f}}{\partial \mathbf{p}}+e \nabla \phi \cdot \frac{\partial \bar{f}}{\partial \mathbf{p}}-\frac{1}{m}(\mathbf{p}-e \mathbf{A} / c) \cdot \nabla \bar{f}
\end{aligned}
$$

In terms of $f(\mathbf{x}, \mathbf{v}, t), \mathcal{K}=m|\mathbf{v}|^{2} / 2$ and (156) becomes

$$
\frac{\partial f}{\partial t}=-\mathbf{v} \cdot \nabla f-\frac{e}{m}\left(\mathbf{E}+\frac{\mathbf{v}}{c} \times \mathbf{B}\right) \cdot \frac{\partial f}{\partial \mathbf{v}}
$$

From the general Hamiltonian (21), with $\mathcal{K}=m|\mathbf{v}|^{2} / 2$, evidently the Vlasov-Maxwell Hamiltonian is

$$
H=\frac{m}{2} \int d \mathbf{x} d \mathbf{v}|\mathbf{v}|^{2} f+\frac{1}{8 \pi} \int d \mathbf{x}\left(|\mathbf{E}|^{2}+|\mathbf{B}|^{2}\right)
$$


and with $[g, h]_{L}$ the bracket of $(\underline{25})-(28)$ becomes the Vlasov-Maxwell bracket

$$
\begin{gathered}
\{F, G\}=\int d \mathbf{x} d \mathbf{v}\left(f\left[F_{f}, G_{f}\right]_{\mathbf{v}}+f\left[F_{f}, G_{f}\right]_{\mathbf{B}}+\frac{4 \pi e}{m} f\left(G_{\mathbf{E}} \cdot \partial_{\mathbf{v}} F_{f}-F_{\mathbf{E}} \cdot \partial_{\mathbf{v}} G_{f}\right)\right) \\
+4 \pi c \int d \mathbf{x}\left(F_{\mathbf{E}} \cdot \nabla \times G_{\mathbf{B}}-G_{\mathbf{E}} \cdot \nabla \times F_{\mathbf{B}}\right) .
\end{gathered}
$$

With this bracket and Hamiltonian, one obtains the usual Vlasov-Maxwell equation as

$$
\frac{\partial f}{\partial t}=\{f, H\}=-[f, \mathcal{K}]_{L}-\frac{e}{m} \mathbf{E} \cdot \frac{\partial f}{\partial \mathbf{v}},
$$

which is equivalent to (57). Similarly, since for this example $\mathbf{D}=\mathbf{E}$, the usual expression for the current is obtained from $\{\mathbf{E}, H\}$, there being no polarization or magnetization contributions, and Faraday's law is given by $\partial \mathbf{B} / \partial t=\{\mathbf{B}, H\}$.

The relativistic Vlasov-Maxwell theory similarly follows with the choice $\mathcal{K}=c \sqrt{|\mathbf{p}-e \mathbf{A} / c|^{2}+m_{0}^{2} c^{2}}$ and the theory can be written in manifestly covariant form [6] , but this will not pursued further here.

\section{B. Guiding Center Drift Kinetic Theory}

A canonical Hamiltonian description for guiding center particle motion was obtained in [6, 7] by applying Dirac constraint theory to Littlejohn's degenerate Lagrangian [15, 16] (with a regularization suggested in [17]) in order to effect a Legendre transformation. The canonical variables of the theory are $\left(\mathbf{x}, q_{4}, \mathbf{p}, p_{4}\right)$ and the particle Hamiltonian, Dirac's primary Hamiltonian for this problem (see e.g. [7, 18]), is

$$
\begin{aligned}
\mathcal{E}\left(\mathbf{x}, q_{4}, \mathbf{p}, p_{4}, \mathbf{E}, \mathbf{B}, \nabla \mathbf{B}\right) & =\mathbf{v}_{g} \cdot\left(\mathbf{p}-e \mathbf{A}^{*} / c\right)+V_{4} p_{4}+e \phi^{*} \\
& =\mathcal{K}\left(\mathbf{p}-e \mathbf{A} / c, q_{4}, p_{4}, \mathbf{E}, \mathbf{B}, \nabla \mathbf{B}\right)+e \phi
\end{aligned}
$$

which is of the form of (44). Here

$$
\begin{aligned}
& \mathbf{A}^{*}=\mathbf{A}+\mathbf{b} m c v_{0} \gamma\left(q_{4} / v_{0}\right) / e, \quad e \phi^{*}=e \phi+\mu|\mathbf{B}|+m\left(q_{4}^{2}+\left|\mathbf{v}_{E}\right|^{2}\right) / 2, \\
& \mathbf{v}_{E}=c(\mathbf{E} \times \mathbf{B}) /|\mathbf{B}|^{2}, \quad \mathbf{b}=\mathbf{B} /|\mathbf{B}|, \\
& \mathbf{v}_{g}=q_{4} \mathbf{B}^{*} /\left(\gamma^{\prime} B_{\|}^{*}\right)+c \mathbf{E}^{*} \times \mathbf{B} / B_{\|}^{*}, \quad V_{4}=e \mathbf{E}^{*} \cdot \mathbf{B}^{*} /\left(m g^{\prime} B_{\|}^{*}\right), \\
& \mathbf{B}^{*}=\nabla \times \mathbf{A}^{*} \quad \text { and } \quad \mathbf{E}^{*}=-\nabla \phi^{*}-\frac{1}{c} \frac{\partial \mathbf{A}^{*}}{\partial t} .
\end{aligned}
$$

The function $\gamma(z)$ is an antisymmetric regularization function with $z=q_{4} / v_{0}$ and $v_{0}$ some constant velocity. The Littlejohn theory is recovered if $\gamma(z)=z$, in which case $q_{4}=v_{\|}$. 
In the regularized theory $\gamma(z) \approx z$ for small $|z|$, but for large $|z|, \gamma$ is bounded so that with $v_{0}>>v_{\text {thermal }}, m v_{0} c \gamma(\infty)<<e|\mathbf{B}| /(\mathbf{b} \cdot \nabla \times \mathbf{b})$, which is accomplished, e.g., by $\gamma=$ $\tanh (z)$.

This theory has an eight-dimensional phase space with the canonical bracket

$$
[g, h]=[g, h]_{\mathbf{p}}+\partial_{q_{4}} g \partial_{p_{4}} h-\partial_{q_{4}} h \partial_{p_{4}} g
$$

which with $\mathcal{K}$ of (62) completes the theory. The appropriate bracket of the form of (25)(28) is obtained with (67), and from $\mathcal{K}$ the functional $K$ can be constructed and thus the Hamiltonian of (21). This bracket and Hamiltonian produces the equations of motion; thus this system is a Hamiltonian field theory. From $K$ the polarization and magnetization are can be obtained straightforwardly. Since expressions are complicated, the reader is referred to [6, 7] for details.

\section{Spin Vlasov-Maxwell}

The nonrelativistic spin Vlasov-Maxwell system is a kinetic theory generalization of the Vlasov-Maxwell system that includes a semiclassical description of spin [19-21]. The Hamiltonian description of this system [21] will be shown to fit within the gauge-free lifiting framework. The spin Vlasov-Maxwell electron distribution function, $f(\mathbf{x}, \mathbf{v}, \mathbf{s}, t)$, contains the internal spin variable $\mathbf{s}=\left(s_{1}, s_{2}, s_{3}\right)$, and satisfies

$$
\frac{\partial f}{\partial t}=-\mathbf{v} \cdot \nabla f+\left[\frac{e}{m}\left(\mathbf{E}+\frac{\mathbf{v}}{c} \times \mathbf{B}\right)+\frac{2 \mu_{e}}{m \hbar c} \nabla(\mathbf{s} \cdot \mathbf{B})\right] \cdot \frac{\partial f}{\partial \mathbf{v}}+\frac{2 \mu_{e}}{\hbar c}(\mathbf{s} \times \mathbf{B}) \cdot \frac{\partial f}{\partial \mathbf{s}},
$$

where $m$ and $e>0$ are the electron mass and charge, respectively, $2 \pi \hbar$ is Planck's constant,

$\mu_{e}=g \mu_{\mathbf{B}} / 2$ is the electron magnetic moment in terms of $\mu_{\mathbf{B}}$, the Bohr magneton, and the electron spin $g$-factor. Equation (68) is coupled to the dynamical Maxwell's equations,

$$
\frac{\partial \mathbf{B}}{\partial t}=-c \nabla \times \mathbf{E}, \quad \frac{\partial \mathbf{E}}{\partial t}=c \nabla \times \mathbf{B}-4 \pi \mathbf{J}
$$

through the current $\mathbf{J}=\mathbf{J}_{f}+c \nabla \times \mathbf{M}$, which has "free" and spin magnetization parts:

$$
\mathbf{J}_{f}:=-e \int d \mathbf{v} d \mathbf{s} \mathbf{v} f \quad \text { and } \quad \mathbf{M}:=-\frac{2 \mu_{e}}{\hbar} \int d \mathbf{v} d \mathbf{s} \mathbf{s} f .
$$

Equations (68) and (69), with (70), are to be viewed classically and consequently a full ninedimensional phase space integration, $d^{9} z=d \mathbf{x} d \mathbf{v} d \mathbf{s}$, is considered for $f$. Spin quantization 
is obtained as an initial condition that constrains $\mathbf{s}$ to lie on a sphere (see [21]). Extension to multiple species is straightforward, so will not be included.

For this system $\mathcal{K}$ is chosen as follows:

$$
\mathcal{K}(\mathbf{v}, \mathbf{s}, \mathbf{B})=\frac{m}{2}|\mathbf{v}|^{2}+\frac{2 \mu_{e}}{\hbar c} \mathbf{s} \cdot \mathbf{B}
$$

however, more general forms are possible. It is not difficult to check that the characteristic equations of $(\underline{68})$ are of the form of (11) with the Poisson bracket $[g, h]=[g, h]_{\mathbf{v}}+[g, h]_{\mathbf{s}}$, where

$$
[g, h]_{\mathbf{s}}=\mathbf{s} \cdot\left(\partial_{\mathbf{s}} g \times \partial_{\mathbf{s}} h\right)
$$

with $\partial_{\mathbf{s}}:=\partial / \partial \mathbf{s}$, and $\mathcal{K}$ given by (71).

Thus the Hamiltonian functional (21) becomes

$$
H[\mathbf{E}, \mathbf{B}, f]=\int d^{9} z\left(\frac{m}{2}|\mathbf{v}|^{2}+\frac{2 \mu_{e}}{\hbar c} \mathbf{s} \cdot \mathbf{B}\right) f+\frac{1}{8 \pi} \int d \mathbf{x}\left(|\mathbf{E}|^{2}+|\mathbf{B}|^{2}\right)
$$

which can be shown to be conserved directly by using the equations of motion, and the bracket of (25)-(28) adapted to the present example is

$$
\begin{aligned}
\{F, G\}_{s V M}=\int d^{9} z f\left(\left[F_{f}, G_{f}\right]_{\mathbf{v}}\right. & +\left[F_{f}, G_{f}\right]_{\mathbf{B}}+\left[F_{f}, G_{f}\right]_{\mathbf{s}} \\
+\frac{4 \pi e}{m}( & \left.\left.F_{\mathbf{E}} \cdot \partial_{\mathbf{v}} G_{f}-G_{\mathbf{E}} \cdot \partial_{\mathbf{v}} F_{f}\right)\right) \\
& +4 \pi c \int d \mathbf{x}\left(F_{\mathbf{E}} \cdot \nabla \times G_{\mathbf{B}}-G_{\mathbf{E}} \cdot \nabla \times F_{\mathbf{B}}\right) .
\end{aligned}
$$

The last term of (74) of $\{,\}_{S V M}$ accommodates the spin, an internal variable; it is not surprising that it has an inner bracket based on the $\mathfrak{s o}(3)$ algebra (e.g. [18]). The remaining terms of (74), (75), and (76) produce the usual terms of Vlasov-Maxwell theory: it is a straightforward exercise to show that Eqs. (68) and (69) are given as follows:

$$
\frac{\partial f}{\partial t}=\{f, H\}_{s V M}, \quad \frac{\partial \mathbf{B}}{\partial t}=\{\mathbf{B}, H\}_{s V M}, \quad \frac{\partial \mathbf{E}}{\partial t}=\{\mathbf{E}, H\}_{s V M} .
$$

This is facilitated by the identity $\int d^{9} z f[g, h]=-\int d^{9} z g[f, h]$, which works for each of $[g, h]_{\mathbf{v}},[g, h]_{\mathbf{B}}$, and $[g, h]_{\mathbf{s}}$. It follows that the polarization $\mathbf{P} \equiv 0$ and $\delta K / \delta \mathbf{B}=-\mathbf{M}$ as given by (70).

In Appendix A2 a direct proof of the Jacobi identity for $\{f, H\}_{s V M}$ is given. 


\section{Monopole Vlasov-Maxwell}

This example differs from the previous ones in that Maxwell's equations are changed to another field theory. In particular, Dirac's theory of electromagnetism [22, 23] with monopole charge will be treated in the lift framework.

For Dirac's theory, (7) is replaced by the particle orbit equations

$$
\dot{\mathbf{x}}=\mathbf{v} \quad \text { and } \quad \dot{\mathbf{v}}=\frac{e}{m}\left(\mathbf{E}+\frac{\mathbf{v}}{c} \times \mathbf{B}\right)+\frac{g}{m}\left(\mathbf{B}-\frac{\mathbf{v}}{c} \times \mathbf{E}\right) .
$$

where $g$ and $e$ are magnetic and electric charges, respectively. The appropriate particle Poisson bracket for insertion into (25) $-(28)$ is

$$
[g, h]_{m}=[g, h]_{\mathbf{v}}+[g, h]_{\mathbf{B}}+[g, h]_{\mathbf{E}}
$$

where

$$
[f, g]_{\mathbf{E}}:=-\frac{g}{m^{2} c} \mathbf{E} \cdot\left(\partial_{\mathbf{v}} f \times \partial_{\mathbf{v}} g\right) .
$$

The particle Hamiltonian is given by $\mathcal{K}=m|\mathbf{v}|^{2} / 2$, just as for Vlasov-Maxwell theory.

In lifting to a kinetic theory there are various kinds of multi-species dynamics, with and without magnetic charge, that could be considered. Here the case of a single species of identical particles that carry both magnetic and electric charges will be developed, along the lines of the quantum fluid theory considered in [24]. Inserting (78) into (25) -(28) and adding a new coupling term to account for the $\mathbf{B}$ that acts as an external force, gives the bracket

$$
\begin{aligned}
& \{F, G\}_{m V M}=\int d \mathbf{x} d \mathbf{v}\left(f\left[F_{f}, G_{f}\right]_{m}\right. \\
& \left.+\frac{4 \pi e}{m} f\left(G_{\mathbf{E}} \cdot \partial_{\mathbf{v}} F_{f}-F_{\mathbf{E}} \cdot \partial_{\mathbf{v}} G_{f}\right)+\frac{4 \pi g}{m} f\left(G_{\mathbf{B}} \cdot \partial_{\mathbf{v}} F_{f}-F_{\mathbf{B}} \cdot \partial_{\mathbf{v}} G_{f}\right)\right) \\
& \quad+4 \pi c \int d \mathbf{x}\left(F_{\mathbf{E}} \cdot \nabla \times G_{\mathbf{B}}-G_{\mathbf{E}} \cdot \nabla \times F_{\mathbf{B}}\right)
\end{aligned}
$$

With Hamiltonian of (58) this bracket yields

$$
\begin{aligned}
\frac{\partial f}{\partial t} & =-\mathbf{v} \cdot \nabla f-\frac{\partial f}{\partial \mathbf{v}} \cdot\left(\frac{e}{m}\left(\mathbf{E}+\frac{\mathbf{v}}{c} \times \mathbf{B}\right)+\frac{g}{m}\left(\mathbf{B}-\frac{\mathbf{v}}{c} \times \mathbf{E}\right)\right) \\
\frac{\partial \mathbf{E}}{\partial t} & =c \nabla \times \mathbf{B}-4 \pi \mathbf{J}_{e} \\
\frac{\partial \mathbf{B}}{\partial t} & =-c \nabla \times \mathbf{E}-4 \pi \mathbf{J}_{m}
\end{aligned}
$$


where

$$
\mathbf{J}_{e}=e \int f v d \mathbf{x} \quad \text { and } \quad \mathbf{J}_{m}=g \int f v d \mathbf{x} .
$$

Thus monopole Vlasov-Maxwell is a Hamiltonian field theory.

One reason for investigating Dirac's model in the present context is to see if the $\nabla \cdot \mathbf{B}=0$ obstruction to Jacobi discussed in Appendix A can be removed. In Appendix A3 the Jacobi identity for $\{F, G\}_{m V M}$ is proved directly, and there it is discovered that the solenoidal character of $\mathbf{B}$ is replaced by $\nabla \cdot(e \mathbf{B}-g \mathbf{E})=0$. Thus, the space of functionals must still be restricted to such fields, as discussed in Appendix A, However, Dirac constraint theory can reduce this to a boundary condition at infinity [25].

Another reason for investigating monopole theories is for their utility in developing numerical algorithms. For example, the Gudunov numerical method for magnetohydrodynamics [26] (see also [27]) exploits a form that allows for $\nabla \cdot \mathbf{B} \neq 0$ that was subsequently shown to be Hamiltonian without the $\nabla \cdot \mathbf{B}=0$ constraint in [1, 3] (see also [28 30]). However, since the monopole theory of this section requires a specific linear combination of $\mathbf{E}$ and $\mathbf{B}$ to be divergence free, adaptations of these methods in not so straightforward. In any event, the reader can rest assured if mononpoles are discovered there still will exist Hamiltonian guiding center and gyrokinetic kinetic theories, obtained with suitable choices for $\mathcal{K}$ with associated generalized polarization and magnetization vectors.

\section{CONCLUSIONS}

The main accomplishment of this work is to describe how a matter model of dynamics can be lifted to a Hamiltonian coupled Vlasov-Maxwell system. En route to this Hamiltonian theory, the general constitutive relations of (30) and (31) or, equivalently, the nonlinear permittivity and permeability operators, as determined by (15), were obtained. These constitutive functionals are very general: as discussed in the paper, $\mathcal{K}$ may contain all derivatives of the fields and may even be global in nature and contain integral operators. From the general constitutive functionals, it was shown how to obtain the usual linear relations. A noncanonical Poisson bracket, another step in the program started in [1 $[3]$, was obtained for this general class of theories.

Four examples were given, including the general class of guiding center kinetic theories of Sec. IVB. This latter example, like all systems that are in the class of variational theories of 
[6], easily was shown to possess the Hamiltonian structure. Thus, the theory of this paper determines the path to follow for obtaining the Hamiltonian formulation of a consistent gyrokinetic theory by making use of the results [31, 32].

Various generalizations are possible. Namely, the extension to many species of different dynamics, relativistic theory other than the Vlasov-Maxwell example that was described, versions where particle matter models have more general finite-dimensional Poisson brackets, are all straightforward. Also, extending the matter dynamics by coupling to other general gauge-free field theories is possible.

Another application of the techniques of this paper will be used in a subsequent work [33] that will treat Hamiltonian perturbation theory in the field theory context. There it will be shown how an exact transformation of the particle (characteristic) equations of VlasovMaxwell equations can be lifted to the kinetic and Maxwell equations, and how this can be used in perturbation theory for infinite-dimensional noncanonical Hamiltonian systems.

In physics there are two ways of constructing new theories. The usual way is to construct an action principle by postulating a Lagrangian density with the desired observables and symmetry group properties. Alternatively one can postulate an energy functional and Poisson bracket, which is essentially the approach of the present paper. With this latter approach, one must prove directly the Jacobi identity $\{\{F, G\}, H\}+\{\{G, H\}, F\}+$ $\{\{H, F\}, G\} \equiv 0$, for all functionals $F, G$, and $H$. Techniques for doing this are not generally known, and this provides one reason for Appendix $\mathrm{A}$.

\section{Appendix A: Direct proof of Jacobi identities}

One term of the original Vlasov-Maxwell Hamiltonian formulation of [2] presented an obstruction to the Jacobi identity [34]. This term was replaced in [12] in order remove this problem, but it was then reported in [3] that the new term also presents an obstruction, viz. that given by (A22) below. One can rescue the Hamiltonian theory by requiring all functionals to depend on fields $\mathbf{B}$ such that $\nabla \cdot \mathbf{B}=0$, but because the orginal program begun in [1, 2] was to construct truly gauge-free field theories in terms of noncanonical Poisson brackets, this taint was a disappointment. The same obstruction appeared in the

context of magnetohydrodynamics [2], but a way to remove it was obtained in [3]. To date, the best fix for the taint of the Vlasov-Maxwell bracket is given in [25] by using Dirac 
constraint theory, which replaces $\nabla \cdot \mathbf{B}=0$ by a boundary condition at infinity.

Appendix A 1 contains the details of the onerous calculation first performed by the author in 1981 (reported in detail here for the first time, as it was originally done), leading to the result of (A22) that appeared in [3]. This is followed in Appendices A2 and A3 by a direct proof of the brackets for the spin and monopole Vlasov-Maxwell theories, respectively.

\section{Jacobi identity for the Vlasov-Maxwell bracket}

For convenience the charge, mass, and a factor of $4 \pi$ are scaled out to obtain the VlasovMaxwell bracket for the fields $f(z, t), E(\mathbf{x}, t)$, and $B(\mathbf{x}, t)$ in the following form:

$$
\begin{aligned}
\{F, G\}= & \int d^{6} z f\left[F_{f}, G_{f}\right]_{c}+f\left[F_{f}, G_{f}\right]_{B}+f\left(G_{E} \cdot \partial_{v} F_{f}-F_{E} \cdot \partial_{v} G_{f}\right) \\
& +\int d^{3} x F_{E} \cdot \nabla \times G_{B}-G_{E} \cdot \nabla \times F_{B} \\
= & :\{F, G\}_{c}+\{F, G\}_{B}+\{F, G\}_{E f}+\{F, G\}_{E B}
\end{aligned}
$$

where $F_{f}:=\delta F / \delta f,[f, g]_{c}:=\nabla f \cdot \partial_{v} g-\nabla g \cdot \partial_{v} f, \partial_{v}:=\partial / \partial v,[f, g]_{B}:=B \cdot\left(\partial_{v} f \times \partial_{v} g\right)$, and $\{F, G\}_{c}$ etc. are obvious from context. Also, note, boldface has been removed since the formulas are busy enough. Since charge can be scaled out in this manner, it is evident that the validity of the Jacobi identity is independent of the sign of the species charge.

Above, the term $\{F, G\}_{B}$, the Marsden-Weinstein term [12], has been separated out because it will be seen to be the source of the failure of Jacobi identity unless $\nabla \cdot \mathbf{B}=0$. Considering the combination $\{F, G\}_{c}+\{F, G\}_{B}$ together would simplify the calculation somewhat.

The Jacobi identity is

$$
\begin{aligned}
\{\{F, G\}, H\} & :=:\left\{\{F, G\}_{c}, H\right\}+\left\{\{F, G\}_{B}, H\right\}+\left\{\{F, G\}_{E f}, H\right\}+\left\{\{F, G\}_{E B}, H\right\} \\
& =\underbrace{\left\{\{F, G\}_{c}, H\right\}_{c}}_{1}+\underbrace{\left.\{f F, G\}_{c}, H\right\}_{B}}_{2}+\underbrace{\left\{\{F, G\}_{c}, H\right\}_{E f}}_{3}+\underbrace{\left.\{F, G\}_{c}, H\right\}_{E B}}_{5} \\
& +\underbrace{\left\{\{F, G\}_{B}, H\right\}_{c}}_{4}+\underbrace{\left\{\{F, G\}_{B}, H\right\}_{B}}_{6}+\underbrace{\left\{\{F, G\}_{B}, H\right\}_{E f}}_{7}+\underbrace{\left.\{f F, G\}_{B}, H\right\}_{E B}}_{8} \\
& +\underbrace{\left\{\{F, G\}_{E f}, H\right\}_{c}}_{9}+\underbrace{\left\{\{F, G\}_{E f}, H\right\}_{B}}_{10}+\underbrace{\left.\{F, G\}_{E f}, H\right\}_{E f}}_{11}+\underbrace{\left\{\{F, G\}_{E f}, H\right\}_{E B}}_{11} \\
& +\underbrace{\left\{\{F, G\}_{E B}, H\right\}_{c}}_{12}+\underbrace{\left\{\{F, G\}_{E B}, H\right\}_{B}}_{14}+\underbrace{\left\{\{F, G\}_{E B}, H\right\}_{E f}}_{15}+\underbrace{\left\{\{F, G\}_{E B}, H\right\}_{E B}}_{15},
\end{aligned}
$$


where the symbol :=: means modulo cyclic permutation; 'cyc' will be used to denote cyclic permutation when only one side is to be permuted.

To prove the Jacobi identity for a generic Poisson bracket of the form $\{F, G\}=\left\langle F_{\psi} \mid \mathcal{J} G_{\psi}\right\rangle$, with cosymplectic operator $\mathcal{J}$, one must calculate the functional derivative $\delta\{F, G\} / \delta \psi$. This derivative has three contributions: one from $F_{\psi}$, one from $\mathcal{J}$, and one from $G_{\psi}$. The first and last give rise to second functional derivatives. Proofs of the Jacobi identity are greatly simplified by the following:

Bracket Theorem ([3]) To prove the Jacobi identity for generic brackets of the form $\{F, G\}=\left\langle F_{\psi} \mid \mathcal{J} G_{\psi}\right\rangle$ one need only consider the explicit dependence of $\mathcal{J}$ on $\psi$ when taking the functional derivative $\delta\{F, G\} / \delta \psi$.

Proof. The formal proof uses the anti-self-adjointness of $\mathcal{J}$ and the self-adjointness of the second functional derivative. With these symmetries it can be shown that all second functional derivative terms cancel.

In what follows $\delta\{F, G\} / \delta \psi \doteq \ldots$ denotes the functional derivative modulo the second derivative terms. Equation (A1) gives

$$
\begin{aligned}
\frac{\delta\{F, G\}_{c}}{\delta f} & \doteq\left[F_{f}, G_{f}\right]_{c}, \quad \frac{\delta\{F, G\}_{c}}{\delta B} \doteq 0, \quad \frac{\delta\{F, G\}_{c}}{\delta E} \doteq 0, \\
\frac{\delta\{F, G\}_{B}}{\delta f} & \doteq\left[F_{f}, G_{f}\right]_{B}, \quad \frac{\delta\{F, G\}_{B}}{\delta B} \doteq \int d v f\left(\partial_{v} F_{f} \times \partial_{v} G_{f}\right), \quad \frac{\delta\{F, G\}_{B}}{\delta E} \doteq 0, \\
\frac{\delta\{F, G\}_{E f}}{\delta f} & \doteq\left(G_{E} \cdot \partial_{v} F_{f}-F_{E} \cdot \partial_{v} G_{f}\right), \quad \frac{\delta\{F, G\}_{E f}}{\delta B} \doteq 0, \quad \frac{\delta\{F, G\}_{E f}}{\delta E} \doteq 0 \\
\frac{\delta\{F, G\}_{E B}}{\delta f} & \doteq 0, \quad \frac{\delta\{F, G\}_{E B}}{\delta B} \doteq 0, \quad \frac{\delta\{F, G\}_{E B}}{\delta E} \doteq 0
\end{aligned}
$$

The following are immediate:

- Term 1 vanishes because $\{F, G\}_{c}$ is Lie-Poisson; i.e., using the first of (A2),$\delta\{F, G\}_{c} / \delta f \doteq\left[F_{f}, G_{f}\right]$, which, when inserted into $\left\{\{F, G\}_{c}, H\right\}_{c}$ and cyclicly permuting, vanishes by virtue of the Jacobi identity of $[,]_{c}$.

- $\underline{\text { Term } 4}$ vanishes by the Bracket Theorem because of the second two equations of (A2).

- $\underline{\text { Term } 12}$ vanishes by the Bracket Theorem because of the second two equations of (A4)

- Terms 13-16 vanish by the Bracket Theorem because $\{F, G\}_{E B}$ has no explicit dependence on $f, E$, or $B$, i.e. because of (A5). 
Remark. One can organize Jacobi identity calculations at the outset by grouping together all like terms that can possibly cancel. For example terms with the same functional derivatives of $F, G$, and $H$ must be considered together. Sometimes other considerations can aid in the grouping of terms. When terms are grouped appropriately, failure of a class of terms to cancel is a proof of the failure of Jacobi. In the heading below $f f f$ means that only function derivatives with respect to $f$ occur, etc.

\section{Term $6(f f f)$}

Using the first equation of (A3) gives

$$
\begin{aligned}
6 & :=: \int f\left[B \cdot\left(\partial_{v} F_{f} \times \partial_{v} G_{f}\right), H_{f}\right]_{B} \\
& :=: \int f B \cdot\left(\partial_{v}\left(B \cdot\left(\partial_{v} F_{f} \times \partial_{v} G_{f}\right)\right) \times \partial_{v} H_{f}\right) \\
& :=: \int f B_{i} B_{r} \epsilon_{i j k} \epsilon_{r s t} \frac{\partial}{\partial v_{j}}\left(\frac{\partial F_{f}}{\partial v_{s}} \frac{\partial G_{f}}{\partial v_{t}}\right) \frac{\partial H_{f}}{\partial v_{k}} \\
& :=: \int f B_{i} B_{r} \epsilon_{i j k} \epsilon_{r s t}\left(\frac{\partial^{2} F_{f}}{\partial v_{j} \partial v_{s}} \frac{\partial G_{f}}{\partial v_{t}} \frac{\partial H_{f}}{\partial v_{k}}+\frac{\partial F_{f}}{\partial v_{s}} \frac{\partial^{2} G_{f}}{\partial v_{j} \partial v_{t}} \frac{\partial H_{f}}{\partial v_{k}}\right) \\
& :=: \int f B_{i} B_{r} \epsilon_{i j k} \epsilon_{r s t}\left(\frac{\partial^{2} F_{f}}{\partial v_{j} \partial v_{s}} \frac{\partial G_{f}}{\partial v_{t}} \frac{\partial H_{f}}{\partial v_{k}}-\frac{\partial H_{f}}{\partial v_{k}} \frac{\partial^{2} F_{f}}{\partial v_{s} \partial v_{j}} \frac{\partial G_{f}}{\partial v_{t}},\right):=: 0
\end{aligned}
$$

where (A7) follows from (A6) by permuting the second term of (A6), shifting the indices according to $s \rightarrow k, k \rightarrow t, t \rightarrow j, j \rightarrow s$, and $i \leftrightarrow r$, and using the antisymmetry of the Levi-Civita symbol.

Note, the above procedure is common in this game and of general utility, so it is recorded in the following:

Lemma 1 If two terms can be made to cancel by permuting one of them, then all terms cancel.

Proof. By writing out all six terms by permuting $F G H \rightarrow G H F \rightarrow H F G$, one observes they cancel in pairs.

Remark. Term 6 vanishes without any assumptions on $B$, i.e. $\nabla \cdot B=0$ is not required.

Term $11(E E f)$ 
Using the first and last equations of (A4) yields

$$
\begin{aligned}
11 & :=: \int f H_{E} \cdot \frac{\partial}{\partial v} \frac{\delta\{F, G\}_{E f}}{\delta f} \\
& :=: \int f H_{E} \cdot \partial_{v}\left(G_{E} \cdot \partial_{v} F_{f}-F_{E} \cdot \partial_{v} G_{f}\right) \\
& :=: \int f\left(H_{E} \cdot \partial_{v}\left(G_{E} \cdot \partial_{v} F_{f}\right)-G_{E} \cdot \partial_{v}\left(H_{E} \cdot \partial_{v} F_{f}\right)\right):=: 0,
\end{aligned}
$$

where the last equality follows because $\operatorname{com}\left(G_{E} \cdot \partial_{v}, H_{E} \cdot \partial_{v}\right)=0$, where com means commutator.

\section{Terms 2 and $5(f f f)$}

Remark. Terms 2 and 5 have been grouped together because both give rise to terms in the Jacobi identity involving $F_{f}, G_{f}$, and $H_{f}$.

Using (A2) in $2+5:=:\left\{\{F, G\}_{c}, H\right\}_{B}+\left\{\{F, G\}_{B}, H\right\}_{c}$ gives

$$
\begin{aligned}
2+5 & :=: \int f B \cdot\left(\partial_{v}\left[F_{f}, G_{f}\right]_{c} \times \partial_{v} H_{f}\right)+f\left[B \cdot\left(\partial_{v} F_{f} \times \partial_{v} G_{f}\right), G_{f}\right]_{c} \\
& =: \int f B_{i} \epsilon_{i j k} \frac{\partial H_{f}}{\partial v_{k}}\left(\left[\frac{\partial F_{f}}{\partial v_{j}}, G_{f}\right]_{c}+\left[F_{f}, \frac{\partial G_{f}}{\partial v_{j}}\right]_{c}\right) \\
& +\int f \epsilon_{i j k}\left(\frac{\partial}{\partial x_{\ell}}\left(B_{i} \frac{\partial F_{f}}{\partial v_{j}} \frac{\partial G_{f}}{\partial v_{k}}\right) \frac{\partial H_{f}}{\partial v_{\ell}}-B_{i} \frac{\partial}{\partial v_{\ell}}\left(\frac{\partial F_{f}}{\partial v_{j}} \frac{\partial G_{f}}{\partial v_{k}}\right) \frac{\partial H_{f}}{\partial x_{\ell}}\right) \\
& :=: \int f B_{i} \epsilon_{i j k}\left(\frac{\partial H_{f}}{\partial v_{k}}\left[\frac{\partial F_{f}}{\partial v_{j}}, G_{f}\right]_{c}+\frac{\partial H_{f}}{\partial v_{k}}\left[F_{f}, \frac{\partial G_{f}}{\partial v_{j}}\right]_{c}+\left[\frac{\partial F_{f}}{\partial v_{j}} \frac{\partial G_{f}}{\partial v_{k}}, H_{f}\right]_{c}\right) \\
& +\int f \epsilon_{i j k} \frac{\partial B_{i}}{\partial x_{\ell}}\left(\frac{\partial F_{f}}{\partial v_{j}} \frac{\partial G_{f}}{\partial v_{k}} \frac{\partial H_{f}}{\partial v_{\ell}}\right) .
\end{aligned}
$$

Upon defining $\mathcal{F}=\partial_{v} F_{f}, \mathcal{G}=\partial_{v} G_{f}$, and $\mathcal{H}=\partial_{v} H_{f}$, and using the Leibniz rule for $[,]_{c}$, Line (A9) can be rewritten as

$$
\int f B_{i} \epsilon_{i j k}\left(\mathcal{H}_{k}\left[\mathcal{F}_{j}, G_{f}\right]_{c}+\mathcal{H}_{k}\left[F_{f}, \mathcal{G}_{j}\right]_{c}+\mathcal{G}_{k}\left[\mathcal{F}_{j}, H_{f}\right]_{c}+\mathcal{F}_{j}\left[\mathcal{G}_{k}, H_{f}\right]_{c}\right):=: 0
$$

where upon permutation the first and fourth terms cancel, as do the second and third. Thus,

$$
2+5:=: \int f \epsilon_{i j k} \frac{\partial B_{i}}{\partial x_{\ell}}\left(\frac{\partial F_{f}}{\partial v_{j}} \frac{\partial G_{f}}{\partial v_{k}} \frac{\partial H_{f}}{\partial v_{\ell}}\right) .
$$

From (A12), the only remaining term is from Line (A10). This term can be rearranged to yield

$$
2+5+\mathrm{cyc}=\int f \nabla \cdot B\left[\left(\partial_{v} F_{f} \times \partial_{v} G_{f}\right) \cdot \partial_{v} H_{f}\right],
$$

which is a consequence of the following: 
Lemma 2 For any three vectors $\mathcal{F}, \mathcal{G}$, and $\mathcal{H}$, and vector field $B$ in $\mathbb{R}^{3}$,

$$
\mathcal{H} \cdot \nabla B \cdot(\mathcal{F} \times \mathcal{G})+\mathcal{F} \cdot \nabla B \cdot(\mathcal{G} \times \mathcal{H})+\mathcal{G} \cdot \nabla B \cdot(\mathcal{H} \times \mathcal{F})=(\nabla \cdot B)(\mathcal{H} \cdot(\mathcal{F} \times \mathcal{G}))
$$

where $\mathcal{H} \cdot \nabla B \cdot \mathcal{C}=\mathcal{H}_{i} \mathcal{C}_{j} \partial_{i} B_{j}$

Remark. Terms 2 and 5 could have been combined with Term 6 . They were considered separately to pinpoint, as will be seen, that they are the sole terms that violate the Jacobi identity without $\nabla \cdot B=0$.

\section{Terms 7 and $10(E f f)$}

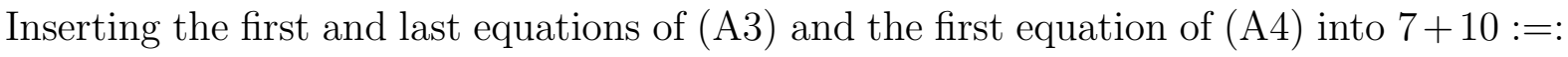
$\left\{\{F, G\}_{B}, H\right\}_{E f}+\left\{\{F, G\}_{E f}, H\right\}_{B}$ gives

$$
\begin{aligned}
& 7+10:=: \int f H_{E} \cdot \partial_{v}\left(B \cdot\left(\partial_{v} F_{f} \times \partial_{v} G_{f}\right)\right) \\
& \left.+f B \cdot\left(\partial_{v}\left(-G_{E} \cdot \partial_{v} F_{f}+F_{E} \cdot \partial_{v} G_{f}\right) \times \partial_{v} H_{f}\right)\right) \\
& :=: \int f H_{E \ell} \partial_{v \ell}\left(B_{i} \epsilon_{i j k} \partial_{v j} F_{f} \partial_{v k} G_{f}\right) \\
& -f B_{i} \epsilon_{i j k}\left(-\partial_{v j}\left(G_{E \ell} \partial_{v \ell} F_{f}\right) \partial_{v k} H_{f}+\partial_{v j}\left(F_{E \ell} \partial_{v \ell} G_{f}\right) \partial_{v k} H_{f}\right) \\
& :=: \int f B_{i} \epsilon_{i j k} H_{E \ell}\left(\partial_{v k} G_{f} \partial_{v \ell} \partial_{v j} F_{f}+\partial_{v j} F_{f} \partial_{v \ell} \partial_{v k} G_{f}\right) \\
& -f B_{i} \epsilon_{i j k}\left(-G_{E \ell} \partial_{v k} H_{f} \partial_{v j} \partial_{v \ell} F_{f}+F_{E \ell} \partial_{v k} H_{f} \partial_{v j} \partial_{v \ell} G_{f}\right) \text {. }
\end{aligned}
$$

Now upon permutation, the first term of (A15) is seen to cancel the second of (A16) and the second term of (A15) cancels the first term of (A16). Thus $7+10:=: 0$.

\section{Terms 3, 8, and $9(E f f)$}

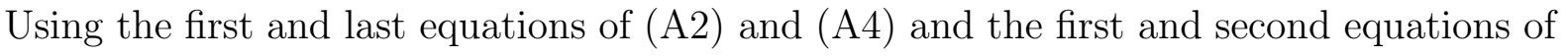
(A3) in $3+9$ gives

$$
3+9:=:-\int f\left(H_{E} \cdot \partial_{v}\left[F_{f}, G_{f}\right]_{c}+\left[H_{E} \cdot \partial_{v} G_{f}, F_{f}\right]_{c}-\left[H_{E} \cdot \partial_{v} F_{f}, G_{f}\right]_{c}\right)
$$

Using Lemma 3 below in (A17) with $C$ equal to $H_{E}$ gives

$$
3+9:=: \int f\left(\nabla \times H_{E}\right) \cdot\left(\partial_{v} F_{f} \times \partial_{v} G_{f}\right) .
$$

Lemma 3 For any vector field $C(x)$ and phase space functions $f$ and $g$,

$$
C \cdot \partial_{v}[f, g]_{c}=\left[C \cdot \partial_{v} f, g\right]_{c}+\left[f, C \cdot \partial_{v} g\right]_{c}+(\nabla \times C) \cdot\left(\partial_{v} g \times \partial_{v} f\right)
$$


Proof. With $\partial_{v i}:=\partial / \partial v_{i}$ and $\partial_{x i}:=\partial / \partial x_{i}$

$$
\begin{aligned}
C \cdot \partial_{v}[f, g]_{c} & =C_{i}\left(\left[\partial_{v i} f, g\right]_{c}+\left[f, \partial_{v i} g\right]_{c}\right) \\
& =\left[C \cdot \partial_{v} f, g\right]_{c}-\left[C_{i}, g\right]_{c} \partial_{v i} f+\left[f, C \cdot \partial_{v} g\right]_{c}-\left[f, C_{i}\right]_{c} \partial_{v i} g \\
& =\left[C \cdot \partial_{v} f, g\right]_{c}-\left(\partial_{v i} f\right)\left(\partial_{v j} g\right) \partial_{x j} C_{i}+\left[f, C \cdot \partial_{v} g\right]_{c}+\left(\partial_{v i} g\right)\left(\partial_{v j} f\right) \partial_{x j} C_{i} \\
& =\left[C \cdot \partial_{v} f, g\right]_{c}+\left[f, C \cdot \partial_{v} g\right]_{c}+\left(\partial_{x j} C_{i}\right)\left(\left(\partial_{v i} f\right)\left(\partial_{v j} g\right)-\left(\partial_{v i} g\right)\left(\partial_{v j} f\right)\right) \\
& =\left[C \cdot \partial_{v} f, g\right]_{c}+\left[f, C \cdot \partial_{v} g\right]_{c}+\left(\partial_{v i} g\right)\left(\partial_{v j} f\right)\left(\partial_{x j} C_{i}-\partial_{x i} C_{j}\right) \\
& =\left[C \cdot \partial_{v} f, g\right]_{c}+\left[f, C \cdot \partial_{v} g\right]_{c}+(\nabla \times C) \cdot\left(\partial_{v} g \times \partial_{v} f\right) \cdot
\end{aligned}
$$

Now consider Term 8

$$
\begin{gathered}
8:=:-\int d^{3} x H_{E} \cdot \nabla \times\left(\int d^{3} v f \partial_{v} F_{f} \times \partial_{v} G_{f}\right) \\
:=:-\int d^{6} z f\left(\nabla \times H_{E}\right) \cdot\left(\partial_{v} F_{f} \times \partial_{v} G_{f}\right) .
\end{gathered}
$$

Equations (A18) and (A21) imply $3+8+9+$ cyc $=0$.

Remark. Observe the terms here, like Terms 7 and 10, are Eff terms. However, they have been grouped separately because there are 'other considerations' as mentioned above. The Terms 7 and 10 vanish with $B$, but the Terms 3, 8, and 9, do not. Thus terms of one kind cannot cancel terms of the other.

Finally, from all of the above, the following is concluded:

Main Theorem ([3] ) For the Vlasov-Maxwell bracket of (A1)

$$
\{\{F, G\}, H\}+\text { cyc }=\int d^{6} z f \nabla \cdot B\left(\partial_{v} F_{f} \times \partial_{v} G_{f}\right) \cdot \partial_{v} H_{f} .
$$

Remark. It is interesting to note that the other constraint, $\nabla \cdot E=4 \pi \rho$, need not be satisfied for the Jacobi identity to hold. It turns out to be a Casimir invariant.

\section{Jacobi identity for the spin Vlasov-Maxwell bracket}

Writing $\{F, G\}_{s V M}=\{F, G\}_{V M}+\{F, G\}_{s}$ and using :=: as defined in Appendix A 1

$$
\begin{aligned}
\left\{\{F, G\}_{s V M}, H\right\}_{s V M}:=:\left\{\{F, G\}_{V M}, H\right\}_{V M}+\left\{\{F, G\}_{s}, H\right\}_{V M}+ \\
+\left\{\{F, G\}_{V M}, H\right\}_{s}+\left\{\{F, G\}_{s}, H\right\}_{s} \\
:=:\left\{\{F, G\}_{s}, H\right\}_{V M}+\left\{\{F, G\}_{V M}, H\right\}_{s},
\end{aligned}
$$


where the second equality follows because of the Jacobi identity for Vlasov-Maxwell (assuming solenoidal $B$ ) and the fact that $\{F, G\}_{s}$ is a Lie-Poisson bracket (see e.g. [4, 35]). Thus it only remains to show that the cross terms cancel, which is facilitated by a the bracket theorem [3] stated in Appendix [A] viz., when functionally differentiating $\{F, G\}_{V M}$ and $\{F, G\}_{s}$, which are needed when constructing the cross terms, one can ignore the second functional derivative terms. Using the symbol $\doteq$ again to denote equivalence modulo the second variation terms,

$$
\begin{aligned}
\frac{\delta\{F, G\}_{V M}}{\delta f} & \doteq\left[F_{f}, G_{f}\right]_{c}+\left[F_{f}, G_{f}\right]_{B}+F_{E} \cdot \partial_{v} G_{f}-G_{E} \cdot \partial_{v} F_{f} \\
\frac{\delta\{F, G\}_{s}}{\delta f} & \doteq\left[F_{f}, G_{f}\right]_{s}
\end{aligned}
$$

while all other needed functional derivatives vanish. Thus

$$
\begin{gathered}
\left\{\{F, G\}_{V M}, H\right\}_{s}:=: \int d^{9} z\left(f\left[\left[F_{f}, G_{f}\right]_{c}+\left[F_{f}, G_{f}\right]_{B}, H_{f}\right]_{s}\right. \\
\left.+f\left[F_{E} \cdot \partial_{v} G_{f}-G_{E} \cdot \partial_{v} F_{f}, H_{f}\right]_{s}\right) \\
\left\{\{F, G\}_{s}, H\right\}_{V M}:=: \int d^{9} z\left(f\left[\left[F_{f}, G_{f}\right]_{s}, H_{f}\right]_{c}+f\left[\left[F_{f}, G_{f}\right]_{s}, H_{f}\right]_{B}\right) \\
\left.+f H_{E} \cdot \partial_{v}\left[F_{f}, G_{f}\right]_{s}\right)
\end{gathered}
$$

The first lines of (A26) and (A27) cancel by virtue of the Jacobi identities for the brackets $[,]_{c, B, s}$ on functions, while the second line of (A26) cancels upon permutation of the second term. Similarly, the second term of (A27) vanishes.

\section{Jacobi identity for the monopole Vlasov-Maxwell bracket}

For this case the Gaussian units of the text are used, i.e. the factors of $4 \pi$ are reinserted and both the usual and monople charges are manifest. Let $\{F, G\}_{m}=\{F, G\}_{V M}+\{F, G\}_{M}$, where

$$
\{F, G\}_{M}=\int d^{6} z f\left[F_{f}, G_{f}\right]_{E}+\frac{4 \pi g}{m} f\left(G_{B} \cdot \partial_{v} F_{f}-F_{B} \cdot \partial_{v} G_{f}\right),
$$

and $[,]_{E}$ is defined by (79). Thus, the Jacobi identity has four terms to consider

$$
\begin{aligned}
\left\{\{F, G\}_{m}, H\right\}_{m} & :=:\left\{\{F, G\}_{V M}, H\right\}_{m}+\left\{\{F, G\}_{M}, H\right\}_{m} \\
& =\underbrace{\left\{\{F, G\}_{V M}, H\right\}_{V M}}_{1}+\underbrace{\left\{\{F, G\}_{M}, H\right\}_{V M}}_{2} \\
& +\underbrace{\left\{\{F, G\}_{V M}, H\right\}_{M}}_{3}+\underbrace{\left\{\{F, G\}_{M}, H\right\}_{M}}_{4},
\end{aligned}
$$


where the symbol :=: is defined in Appendix A 1.

\section{Term 1}

From the [3] (cf. Appendix A 1)

$$
\left\{\{F, G\}_{V M}, H\right\}_{V M}+\mathrm{cyc}=\frac{e}{m^{2}} \int d^{6} z f \nabla \cdot B\left[\left(\partial_{v} F_{f} \times \partial_{v} G_{f}\right) \cdot \partial_{v} H_{f}\right] .
$$

As in Appendix A 1, $\delta\{F, G\} / \delta \psi \doteq \ldots$ denotes the functional derivative modulo the second derivative terms. The following will be needed:

$$
\begin{aligned}
& \frac{\delta\{F, G\}_{V M}}{\delta f} \doteq\left[F_{f}, G_{f}\right]_{c}+\left[F_{f}, G_{f}\right]_{B}+\frac{4 \pi e}{m}\left(G_{E} \cdot \partial_{v} F_{f}-F_{E} \cdot \partial_{v} G_{f}\right) \\
& \frac{\delta\{F, G\}_{M}}{\delta f} \doteq\left[F_{f}, G_{f}\right]_{E}+\frac{4 \pi g}{m}\left(G_{B} \cdot \partial_{v} F_{f}-F_{B} \cdot \partial_{v} G_{f}\right) \\
& \frac{\delta\{F, G\}_{V M}}{\delta B} \doteq \frac{e}{m^{2}} \int d^{3} v f \partial_{v} F_{f} \times \partial_{v} G_{f}, \quad \frac{\delta\{F, G\}_{V M}}{\delta E} \doteq 0 \\
& \frac{\delta\{F, G\}_{M}}{\delta E} \doteq-\frac{g}{m^{2}} \int d^{3} v f \partial_{v} F_{f} \times \partial_{v} G_{f}, \quad \frac{\delta\{F, G\}_{M}}{\delta B} \doteq 0 .
\end{aligned}
$$

The Poisson bracket that generates the ' $v \times$ ' part of the generalized Lorentz force is $[f, g]=[f, g]_{c}+[f, g]_{B}+[f, g]_{E}$. Because of

$$
\left[[f, g]_{E}, h\right]_{E}+\text { cyc }=0 \text { and } \quad\left[[f, g]_{E}, h\right]_{B}+\left[[f, g]_{B}, h\right]_{E}+\text { cyc }=0 \text {. }
$$

the following holds:

$$
\begin{aligned}
{[[f, g], h]+\text { cyc } } & =\left[[f, g]_{c}, h\right]_{B}+\left[[f, g]_{B}, h\right]_{c}+\left[[f, g]_{c}, h\right]_{E}+\left[[f, g]_{E}, h\right]_{c}+\text { cyc } \\
& =(e \nabla \cdot B-g \nabla \cdot E)\left[\left(\partial_{v} f \times \partial_{V} g\right) \cdot \partial_{V} h\right] / m^{2}
\end{aligned}
$$

The first term of the above is the source of the RHS of (A30).

Now consider the remaining terms of (A29).

\section{Term 4}

Equations (A32) and (A28) give

$$
\begin{gathered}
\left\{\{F, G\}_{M}, H\right\}_{M}:=: \int d^{6} z f\left[\left[F_{f}, G_{f}\right]_{E}+\frac{4 \pi g}{m}\left(G_{B} \cdot \partial_{v} F_{f}-F_{B} \cdot \partial_{v} G_{f}\right), H_{f}\right]_{E} \\
+\frac{4 \pi g}{m} f H_{B} \cdot \partial_{v}\left(\left[F_{f}, G_{f}\right]_{E}+\frac{4 \pi g}{m}\left(G_{B} \cdot \partial_{v} F_{f}-F_{B} \cdot \partial_{v} G_{f}\right)\right) .
\end{gathered}
$$

Equation (A37) has three kinds of terms. Consider first the $f f f$-terms:

$$
\begin{gathered}
\int d^{6} z f\left[\left[F_{f}, G_{f}\right]_{E}, H_{f}\right]_{E}=\frac{g^{2}}{m^{4}} \int d^{6} z f E \cdot\left(\partial_{v}\left(E \cdot\left(\partial_{v} F_{f} \times \partial_{v} G_{f}\right)\right) \times \partial_{v} H_{f}\right) \\
=\frac{g^{2}}{m^{4}} \int d^{6} z f \epsilon_{i j k} \epsilon_{r s t} E_{i} E_{r} \partial_{v k} H_{f}\left(\partial_{v j} \partial_{v s} F_{f} \partial_{v t} G_{f}\right. \\
\left.+\partial_{v j} \partial_{v t} G_{f} \partial_{v s} F_{f}\right)
\end{gathered}
$$


Upon permutation and reindexing, the two terms of (A38) cancel. This, of course, follows immediately from (A35) - the above serves as a proof that $\left[[f, g]_{E}, h\right]_{E}+$ cyc $=0$. Next consider the $B B f$-term:

$$
\frac{16 \pi^{2} g^{2}}{m^{4}} \int d^{6} z f H_{B} \cdot \partial_{v}\left(G_{B} \cdot \partial_{v} F_{f}-F_{B} \cdot \partial_{v} G_{f}\right)
$$

This vanishes upon permutation because $H_{B} \cdot \partial_{v}$ and $F_{B} \cdot \partial_{v}$ commute. Now all that remains of Term 4 is the $B f f$-term:

$$
\frac{4 \pi g}{m} \int d^{6} z f\left[\left(G_{B} \cdot \partial_{v} F_{f}-F_{B} \cdot \partial_{v} G_{f}, H_{f}\right]_{E}+f H_{B} \cdot \partial_{v}\left[F_{f}, G_{f}\right]_{E}\right.
$$

This term is of the same form as the EFF term of the MV-bracket (terms 7 and 10), and vanishes for the same reason. Therefore, Term 4 vanishes.

Now consider the two cross terms.

\section{Terms 2 and 3}

Term 2 is

$$
\begin{aligned}
\left\{\{F, G\}_{M}, H\right\}_{V M} & :=: \int d^{6} z f\left[\left[F_{f}, G_{f}\right]_{E}+\frac{4 \pi g}{m}\left(G_{B} \cdot \partial_{v} F_{f}-F_{B} \cdot \partial_{v} G_{f}\right), H_{f}\right]_{c} \\
& +f\left[\left[F_{f}, G_{f}\right]_{E}+\frac{4 \pi g}{m}\left(G_{B} \cdot \partial_{v} F_{f}-F_{B} \cdot \partial_{v} G_{f}\right), H_{f}\right]_{B} \\
& +\frac{4 \pi e}{m} f H_{E} \cdot \partial_{v}\left(\left[F_{f}, G_{f}\right]_{E}+\frac{4 \pi g}{m}\left(G_{B} \cdot \partial_{v} F_{f}-F_{B} \cdot \partial_{v} G_{f}\right)\right) \\
& +\frac{e}{m} f \partial_{v} H_{f} \cdot \frac{g}{m^{2}} \int d^{3} v f \partial_{v} F_{f} \times \partial_{v} G_{f} \\
& -4 \pi \int d^{3} x \nabla \times H_{B} \cdot \frac{g}{m^{2}} \int d^{3} v f \partial_{v} F_{f} \times \partial_{v} G_{f},
\end{aligned}
$$

while Term 3 is

$$
\begin{aligned}
\left\{\{F, G\}_{V M}, H\right\}_{M}:= & : \int d^{6} z f\left[\left[F_{f}, G_{f}\right]_{c}+\left[F_{f}, G_{f}\right]_{B}\right. \\
& \left.+\frac{4 \pi e}{m}\left(G_{E} \cdot \partial_{v} F_{f}-F_{E} \cdot \partial_{v} G_{f}\right), H_{f}\right]_{E} \\
+ & \frac{4 \pi g}{m} f H_{B} \cdot \partial_{v}\left(\left[F_{f}, G_{f}\right]_{c}+\left[F_{f}, G_{f}\right]_{B}\right. \\
& \left.+\frac{4 \pi e}{m}\left(G_{E} \cdot \partial_{v} F_{f}-F_{E} \cdot \partial_{v} G_{f}\right)\right) \\
- & \frac{g}{m} f \partial_{v} H_{f} \cdot \frac{e}{m^{2}} \int d^{3} v f \partial_{v} F_{f} \times \partial_{v} G_{f} .
\end{aligned}
$$

Upon comparing Terms 2 and 3 some cancellations are immediate. 
- Using (A36), 1 st term of (A41) +1 st term of (A46) gives $-\frac{g}{m^{2}}(\nabla \cdot E)\left(\partial_{v} F_{f} \times \partial_{v} G_{f}\right)$. $\partial_{v} H_{f}$

- Using (A35), 1st term of (A42) + 2nd term of (A46) $=0$

- Lines $(\underline{\mathrm{A} 44})+(\underline{\mathrm{A} 50})=0$

- The terms of (A49) vanish because $H_{B} \cdot \partial_{v}$ and $G_{E} \cdot \partial_{v}$ commute. Likewise the last two terms of (A43)

Applying the following:

Lemma For any vector field $C(x)$ and phase space functions $f$ and $g$,

$$
C \cdot \partial_{v}[f, g]_{c}=\left[C \cdot \partial_{v} f, g\right]_{c}+\left[f, C \cdot \partial_{v} g\right]_{c}+m^{-1}(\nabla \times C) \cdot\left(\partial_{v} g \times \partial_{v} f\right),
$$

which is not difficult to prove, to the last terms of (A41) + Line (A45) + the last first term of $(\underline{\mathrm{A} 48 \mathrm{~B}})=0$.

There are six remaining terms. The last two terms of (A42) cancel the last term of (A48), and the first term of (A43) cancels the two terms of (A47). Thus in this case, the obstruction becomes

$$
\begin{aligned}
& \left\{\{F, G\}_{m V M}, H\right\}_{m V M}+\mathrm{cyc}= \\
& \frac{1}{m^{2}} \int d^{6} z f(e \nabla \cdot B-g \nabla \cdot E)\left(\partial_{v} F_{f} \times \partial_{v} G_{f}\right) \cdot \partial_{v} H_{f},
\end{aligned}
$$

and it is concluded that the Jacobi identity still requires a solenoid constraint on $e B-g E$.

Upon transforming to new variables $\tilde{e} \tilde{E}=e E+g B$ and $\tilde{e} B=e B-g E$, where $\tilde{e}^{2}=e^{2}+g^{2}$, reproduces the Poisson bracket for Vlasov-Maxwell theory, which is possible for this single species case of Dirac's theory. In retrospect, the existence of this transformation precludes the necessity for the proof of the Jacobi identity; however, consistent with the goal of this entire appendix, viz. to demonstrate techniques of general utility rather than to present the most efficient proofs, we retain it here.

\section{ACKNOWLEDGMENT}

I would like to thank Alain Brizard, Cristel Chandre, Emanuele Tassi, and Michel Vittot for their continued interest in this subject, their encouragement, and for many fruitful discussions. I would also like to thank Iwo Bialynicki-Birula for helpful correspondence and Loic 
de Guillebon for his comments on an earlier draft of this manuscript. Supported by U.S. Dept. of Energy Contract \# DE-FG05-80ET-53088.

[1] P. J. Morrison and J. M. Greene, Phys. Rev. Lett. 45, 790 (1980), E 48, 569 (1982).

[2] P. J. Morrison, Phys. Lett. A 80, 383 (1980).

[3] P. J. Morrison, in Mathematical Methods in Hydrodynamics and Integrability in Dynamical Systems, edited by M. Tabor and Y. Treve (New York: Am. Inst. Phys., 1982), vol. 88, pp. $13-46$.

[4] P. J. Morrison, Rev. Mod. Phys. 70, 467 (1998).

[5] D. Pfirsch, Z. Naturforsch. 39a, 1 (1984).

[6] D. Pfirsch and P. J. Morrison, Phys. Rev. 32A, 1714 (1985).

[7] D. Pfirsch and P. J. Morrison, Phys. Fluids 3B, 271 (1991).

[8] R. G. Littlejohn, J. Math. Phys. 20, 2445 (1979).

[9] P. J. Morrison, Phys. Plasmas 12, 058102 (2005).

[10] M. Born and L. Infeld, Proc. Roy. Soc. 147A, 522 (1934).

[11] I. Bialynicki-Birula and Z. Bialynicki-Birula, Quantum Electrodynamics (Oxford: Pergamon Press, 1975).

[12] J. E. Marsden and A. Weinstein, Phys. 4D, 394 (1982).

[13] I. Bialynicki-Birula, J. C. Hubbard, and L. A. Turski, Physica 128A, 509 (1984).

[14] S. Ichimaru, Basic Principles of Plasma Physics (Benjamin, Frontiers in Physics, 1973).

[15] R. G. Littlejohn, Phys. Fluids 24, 1730 (1981).

[16] R. G. Littlejohn, J. Plasma Phys. 29, 111 (1983).

[17] H. K. Wimmel, Phys. Scripta 29, 141 (1984).

[18] E. C. G. Sudarshan and N. Makunda, Classical Dynamics : A Modern Perspective (John Wiley \& Sons, New York, 1974).

[19] G. Brodin, M. Marklund, A. Zamanian, J. Ericsson, and P. L. Mana, Phys. Rev. Lett. 101, $245002(2008)$.

[20] G. Brodin, M. Marklund, and G. Manfredi, Phys. Rev. Lett. 100, 175001 (2008).

[21] M. Marklund and P. J. Morrison, Phys. Lett. A 375, 23622365 (2011).

[22] P. A. M. Dirac, Proc. Roy. Soc. A 133, 1 (1931). 
[23] P. A. M. Dirac, Phys. Rev. 74, 817 (1948).

[24] I. Bialynicki-Birula and Z. Bialynicki-Birula, Phys. Rev. 3, 2410 (1971).

[25] C. Chandre, L. de Guillebon, A. Back, E. Tassi, and P. J. Morrison, preprint arXiv:1205.2347v1 (2012).

[26] S. K. Gudunov, Dokl. Akad. Nauk. 139, 521 (1961).

[27] P. J. Dellar, J. Comp. Phys. 172, 392 (2001).

[28] T. Andreussi, P. J. Morrison, and F. Pegoraro, Plasma Phys. Cont. Fusion 52, 055001 (2010).

[29] T. Andreussi, P. J. Morrison, and F. Pegoraro, Phys. Plasmas 19, 052102 (2012).

[30] T. Andreussi, P. J. Morrison, and F. Pegoraro, Phys. Plasmas (2012).

[31] D. Pfirsch and D. Correa-Restrepo, J. Plasma Phys. 70, 719 (2004).

[32] D. Correa-Restrepo and D. Pfirsch, J. Plasma Phys. 70, 757 (2004).

[33] P. J. Morrison, M. Vittot, and L. de Guillebon, preprint arXiv:1212.3007 [physics.plasm-ph] (2012).

[34] A. Weinstein and P. J. Morrison, Phys. Lett. 86A, 235 (1981).

[35] J. E. Marsden and T. S. Ratiu, Introduction to Mechanics and Symmetry (New York: Springer, 1999). 